\title{
Semantics without Toil? Brady and Rush Meet Halldén
}

\author{
Lloyd Humberstone*
}

Received: 23 August 2018 / Accepted: 3 November 2018

Abstract: The present discussion takes up an issue raised in Section 5 of Ross Brady and Penelope Rush's paper 'Four Basic Logical Issues' concerning the (claimed) triviality - in the sense of automatic availability - of soundness and completeness results for a logic in a metalanguage employing at least as much logical vocabulary as the object logic, where the metalogical behaviour of the common logical vocabulary is as in the object logic. We shall see - in Propositions 4.5-4.7 - that this triviality claim faces difficulties in the face of Halldén incompleteness, for essentially the same reasons that Halldén thought this phenomenon raised semantic difficulties for the modal logics of C. I. Lewis exhibiting it. To counter any inclination to dismiss the phenomenon as providing at best a marginal range of counterexamples to the triviality claim, a Postscript assembles some reminders of the extent of - and the varied considerations favouring - Halldén incompleteness.

Keywords: Nonclassical logic; semantic completeness; homophonic model theory; Halldén completeness; choice of meta-logic.

${ }^{*}$ Monash University

Department of Philosophy and Bioethics, Monash University, Victoria 3800, Australia.

凶 lloyd.humberstone@monash.edu

(C) The Author. Journal compilation (c) The Editorial Board, Organon F. 


\section{Introduction and initial difficulties}

The material under (2) of the following quotation from Section 5 (entitled "Classical meta-logic versus nonclassical meta-logic") of (Brady and Rush 2009) provides the stimulus for the present discussion; the rest of the quotation is included to provide context. Note that letters from the range $p, q, r, \ldots$ are used as sentence letters (propositional variables) while $A, B, \ldots$ are metalinguistic variables over formulas of arbitrary complexity. The bibliographical references have been adjusted to match the style used here:

(1) Intensional logic requires an intensional semantics.

This has been strongly argued by (Meyer and Routley 1977) and in the appendix of (Brady 2003), rejecting extensional reduction in favor of a semantics that more directly captures the meaning of the object logic. But, what nonclassical meta-logic is appropriate? Does this mean that it is the same logic as that of the object language? This leads us to the second point.

(2) Trivial semantics (or toil-less semantics) is just that.

Meyer and Sylvan (né Routley) have also made this point, in conversation. Here, such a semantics interprets each connective according to the logic of the object language, as follows:

$I(p)$ is assigned $T$ or not assigned $T$, for each $p$.

$I(\sim A)=T$ iff not $(I(A)=T){ }^{1}$

$I(A \& B)=T$ iff $I(A)=T$ and $I(B)=T$.

$I(A \vee B)=T$ iff $I(A)=T$ or $I(B)=T$.

$I(A \rightarrow B)=T$ iff, if $I(A)=T$ then $I(B)=T$.

A formula $A$ is valid iff $I(A)=T$, for all assignments to its variables.

The Soundness and Completeness Theorems are then trivial. For completeness, for a nontheorem $N, I(N)=T$ would fail as a result of the failure of the corresponding metalogical law, applied to totally unconstrained assignments to its variables.

\footnotetext{
${ }^{1}$ In fact what appears in (Brady and Rush 2009) at this point is " $I(\sim A)=T$ iff not $-(I(A)=T)$ ", but the "-" is a typo (presumably intended as a hyphen linking "not" and " $(I(A)=T)$ ". Four lines down, italics are added here for the word "valid", since the statement is intended as a definition.
}

Organon F 26 (3) 2019: 340-404 
For the remainder of this section and the following two sections, we examine aspects - both technical and philosophical - of this passage and the background against which it is set. Readers keen to get to the encounter with Halldén promised in the subtitle, and described in the Abstract as threatening the above triviality claim, may like to skim hastily over that material to arrive at the start of Section 4, in which the crucial observations appear (Propositions 4.5-4.7). At the other end of the patience spectrum, those wanting to take the scenic route over the terrain of Sections 1-3 should consult the 'longer notes' on those sections collected in the Appendix as references to them appear in the main body of the paper.

In what follows, the notation " $\&$ ", " $\sim$ ", for conjunction and negation will be used only when quoting Brady and Rush, and will otherwise be replaced by " $\wedge$ " and " $\neg "$. The letter "I" used by Brady and Rush is intended to suggest interpretation, and as we see, interpretations here are taken to be assignments of truth-values, of which at least one is $T$. Since this is indeed the only value mentioned in the above inductive definition of truth on an interpretation, there is nothing to be lost in replacing the functional notation with a less committal notation and writing " $I \models A$ " in place of " $I(A)=T$ ".

Remark 1.1 Nothing to be lost... and in fact, there may be something to be gained by making this replacement: namely the avoidance of extraneous complications. Such complications include the following, which arose in the course of giving a semantics along these lines in (Humberstone 1996) - an enterprise not there regarded as trivial - for the case of $\square$ understood as expressing something like metaphysical necessity. One issue here is that if identities hold of necessity if at all, then stipulating that $I(\square A)=T$ if and only if necessarily $I(A)=T$ is in danger of committing us to: $I(\square A)=T$ if and only if $I(A)=T$, for any $I$. (Instead of $I(A)$, (Humberstone 1996) has " $v(A)$ " - " $v$ " for valuation; see (Humberstone 1996) for a reaction to the danger. No more will be said about (Humberstone 1996) in what follows, except to point to some background clarification in note 3 . The paper was written before the difficulties for homophonic model theory presented in Section 4 were noticed, and the fact that the main one stressed in that Section, connected with Halldén incompleteness, does not arise for the modal logic in play there does not guarantee that similar problems do not arise.) One might avoid this result by saying, in an informal meta-metalanguage, that " $I(\square A)$ ", for a fixed $I$ and $A$, is somehow not a rigid designator and so the necessity of identities 
does not disallow contingent identity statements in this case, or again that one rejects this principle anyway, or ... But it seems better not to get into debates about identity when the identity predicate of the object language is not even under discussion. (It must be conceded that a shadow of the worry remains after the expulsion of "=": how does it get to be contingent, for a fixed $I$ and $A$, whether or not $I \models A$ ? Note also that it is not only in the logic of metaphysical necessity but also in the logic of vagueness that identifying the truth on an interpretation of a vague sentence with a something's being identical with something may raise difficulties for anyone thinking that identity statements can be vague - (Evans 1978) and all that.) Though we continue to use the $I(A)=T$ notation when dealing with the explicit discussion in (Brady and Rush 2009), we return to the pitfalls invited by doing so in Remark 4.4 below.

Presumably it would not be appropriate to respond to the example described in Remark 1.1 by saying that a necessity operator was not one of the connectives explicitly listed in the above passage from Brady and Rush: the cases listed are there by way of example, the intention being to illustrate a general point arising with conditions for arbitrary sentence connectives; and in any case, though considerable play is made in Section 4 with modal examples, there is no special need to think of the $\square$ figuring in them as representing any kind of necessity. Perhaps the authors intend also to include the case of quantifiers in the object language, but since their discussion is restricted to sentential logic, let us follow their lead. But we do need quantifiers in the metalanguage (and this is the reason the Abstract of the present discussion talks about the metalanguage having at least the logical vocabulary of the object language), since universal quantification is used tacitly over the $A, B$ in the conditions governing $\sim, \&, \vee$ and $\rightarrow$ and then explicitly in the definition of validity near the end of the passage. ${ }^{2}$ Concerning the latter definition, a further corrective adjustment to the text like those alluded to in note 1 could have been made and explained there, but it raises an issue that deserves slightly greater attention than such those typographical matters. So that we don't have even the appearance of a definition in which a variable (here "I") appears free in the definiens but not the definiendum (" $A$ is valid"), it would

\footnotetext{
${ }^{2}$ Adding an extra layer of complexity, we need, not only quantification over interpretations for validity but then quantification over valid formulas (or more generally, valid inferences) in the definitions of soundness and completeness - as remarked in (Williamson 2017, 159) - to say nothing of (typically) existential quantification for the notion of provability.
}

Organon F 26 (3) 2019: 340-404 
have been clearer to write "for all assignments $I$ to its variables" (where this used of variables is to the propositional variables or sentence letters of the object language), but perhaps the authors did not do this because they wanted to think of $I$ as interpreting all formulas and not just as an assignment of truth-values to the sentence letters. There are two ways of handling this issue commonly encountered. One of them makes a notational distinction between the assignment to the atomic expressions in question (here, the sentence letters), $I$, as it might be, and then uses the inductive definition provided by the four conditions just recalled to extend this to an interpretation function $I^{+}$, say, applying to all expressions (here, all formulas). The other is just to say that an interpretation $I$ is any function satisfying the conditions given, and to note in passing that what $I$ does to the atomic expressions in fact determines what it does to all expressions. On the latter approach, which I assume is not something that would raise an objection from Brady and Rush, the definition of validity would just be that a formula $A$ is valid iff for all interpretations $I$, $I(A)=T$ (or, perhaps preferably, for the reasons given in Remark 1.1: iff for all interpretations $I, I \models A$ ). We return in Section 2 to what is being assumed about this "iff" in the possibly non-classical metalanguage is supposed to be.

Setting that question to one side for the moment, what we have in the quoted passage is an example of homophonic model-theoretic semantics - homophonic in the sense that items of the logical vocabulary of the object language - in the present case, various sentence connectives - are paired up with corresponding items from the metalanguage, which, in the case in which the metalanguage literally includes the object language, will indeed 'sound the same'. The "model-theoretic" part of the description may seem somewhat overblown for the deployment of interpretations lacking internal structure, but it enjoys a certain currency for marking the relativity of truth to something or other-models, valuations or interpretations (here $I$ ), contrasting with the case of homophonic semantics in the (absolute) truth-theoretic tradition for an interpreted language, in which there is no question of varying the interpretation concerned. ${ }^{3}$ If in such model-theoretic semantics there is also an idle relativity to a further parameter - such as a time or a world, or more neutrally, a point in a Kripke (or similar) model - we can usefully follow the example of

\footnotetext{
${ }^{3}$ See (Humberstone 1996) for further discussion and references. A better name for present purposes might be interpretational semantics, as in (Evans 1976), but this would be distracting below, because of the use by Brady and Rush of the phrase "interpretational metalogic" whose exact significance, it will be suggested below, is at best elusive. Further references to homophonic semantics for intensional languages can be found in (Bräuner 2002, 364), though most of this is of the truth-theoretic rather than the model-theoretic kind.
}

Organon F 26 (3) 2019: 340-404 
(Williamson 2017) (and its precursor and companion pieces (Williamson 2011) and (Williamson 2014)) in speaking of a quasi-homophonic treatment in this case, as well as being more fine-grained in the application of this vocabulary to the individual clauses in the inductive definition of (interpretation-relative) truth. Thus the Kripke semantics for modal logic treats the Boolean connectives quasi-homophonically (the world parameter being idle) but the modal operators heterophonically, since they are treated, not by modal clauses in the semantic metalanguage but by (typically restricted) quantification in the metalanguage. Indeed, the introduction of the "quasi-homophonic" terminology is only called for by such semantic accounts in which at least some vocabulary is treated heterophonically, since if the ancillary parameter(s) is/are idle in every semantic clause then we can repackage them along with what we were previously thinking of as an interpretation into something and now think of this whole caboodle - for example it can be an interpretation (or model) paired with a world, to recall the case just mentioned - trading in quasi-homophonic semantic for homophonic semantics.

It should be stressed that not every attempt to use the same logic at the meta-level in discussing a certain object logic, even when the discussion concerns the model theory for that logic, is a case of homophonic model-theoretic semantics. For example, in the 1970s, W. Veldman and H. de Swart modified, respectively, Kripke models (for intuitionistic logic) and Beth trees to give structures for which (they claimed) intuitionistically acceptable completeness proofs could be provided. The references can be found in (Dummett 1977, $\S 5.7) ;{ }^{4}$ for scepticism about such ventures, see (McCarty 2008) and references therein. A famous example from the following decade appeared in (Meyer 1985) where a relevant-logically acceptable completeness proof w.r.t. the (Routley-Meyer) heterophonic model-theoretic semantics is sought for the $\operatorname{logic} \mathbf{R} .{ }^{5}$ More specifically, the model theory in both the intuitionistic and relevant logical cases treats conjunction and disjunction quasi-homophonically but implication and negation heterophonically. (Here in the case of relevant logic we have the "Australian plan' in mind, with "*" function treatment of nega-

\footnotetext{
${ }^{4}$ This is one point at which the discussion in the first and second editions of (Dummett 1977) differ, though both writers are touched on in both editions and the difference is not pertinent to the present theme.

${ }^{5}$ The idea is that although this is not homophonic model theory, since the logic metalanguage is also (albeit quantified) $\mathbf{R}$ we have what we might call an 'isological' semantic treatment. It would be nicer if we could say homological here, but that term is already taken, though even if it weren't, we wouldn't be able to mirror the homophonic/heterophonic contrast directly since heterological is likewise already spoken for - in Grelling's Paradox (and opposed there to autological).
}

Organon F 26 (3) 2019: 340-404 
tion. ${ }^{6}$ Similarly, in (Girard and Weber 2018), summarised more fully in the longer note 'Bacon and others' on Section 2 in the Appendix, the non-modal logical vocabulary is treated homophonically while the modal vocabulary is treated heterophonically (in a contraction-free metalogic). (Meyer 1985) deserves to be counted as famous, despite its never having been published (other than as an ANU research report), on the strength of the frequency which its description of the aim of the widespread practice - from which (Meyer 1985) itself departs - of using classical logic in the semantic metalanguage for relevant logic. The aim was said to be to "preach to the Gentiles in their own tongue", a description whose charm becomes especially apparent when we recall Meyer's own early days as a Christian missionary (in Japan). Among the items in the bibliography of the present paper for independent reasons in which this turn of phrase is quoted are (Read 1988) (as well as (Aberdein and Read 2009)) and (Weber, Badia and Girard 2016) - though there may be more. Read's own 'Scottish Plan' for the semantic of relevant logic (Read $1988, \S 7.3$ and $\S \S 8.4-8.6)$ is, by contrast, not only metalogically relevant but treats all vocabulary homophonically. ${ }^{7}$ (Priest 2006, 98) thinks that the use of a non-classical metalogic is not just permissible but is be mandated by considerations of integrity, writing that intuitionists and dialetheists take themselves "to be giving an account of the correct behaviour of certain logical particles. Is it to be supposed that their account of this behaviour is to be given in a way that they take to be incorrect? Clearly not. The same logic must be used

\footnotetext{
${ }^{6}$ See (Meyer and Martin 1986) for the contrast between this and the (Dunn-Belnap or) 'American plan' approach. Brady and Rush touch on both in their discussion but the treatment of the latter on p. 495 is somewhat garbled: we have clauses such as - to illustrate with the case of negation $-I(\sim A)=T$ iff $I(A)=F$ and $I(\sim A)=F$ iff $I(A)=T$, and it is remarked that when $A$ and $B$ are "classically evaluated, that is, $I(A)$ and $I(B)$ each take only one value, that is, $T$ or $F$ ", then it "clearly follows that $\sim A, A \& B$, and $A \vee B$ are also classically evaluated." But the uniqueness of the value is built into the functional notation from the start, and if $I$ is going to be a function, it should be assigning subsets rather than elements of $\{T, F\}$ to the formulas with things like " $I(A)=F$ ", as just seen, replaced with " $F \in I(A)$ " etc. For the first-degree entailments, Belnap and Dunn dip a toe into homophonic waters in $\S 2.5$ of (Belnap and Dunn 1981), reproduced as $\S 80.25$ of (Anderson, Belnap and Dunn 1992).

${ }^{7}$ Semantic consequence is handled by modalizing the relevant logic, so at first blush the approach seems truth-theoretic rather than model-theoretic, in the absence of anything like quantification over interpretations, though Read thinks otherwise: first new paragraph on p.163. Whatever the right view is of this issue, the enterprise is highly engaging. The idea is to have relevance fall out of the idea of necessary truth-preservation rather than be tacked on as an additional filter, by taking truth preservation as a matter of not having (as I shall put it) true premisses while having a false conclusion, where this "while" is understood as a metalinguistic deployment of the intensional ('fusion') rather than the extensional conjunction which relevant logic is famous for distinguishing - more widely encountered, perhaps, in subsequent years as the multiplicative rather than the additive version of conjunction from linear logic. Some further aspects of (Read 1988) were touched on in the review (Humberstone 1991).
} 
in both 'object theory' and 'metatheory'." ${ }^{8}$ For more on this, see the longer note 'Dummett and others' in the Appendix.

These heterophonic uses of non-classical logic in the metatheory of a corresponding non-classical logic are far from toil-free - or 'toil-less' as it is put in the quoted passage - in which Brady and Rush are certainly not suggesting that the only way to use a non-classical meta-logic is in the is this, as they say, trivial semantics. The gist of the qualms raised below will not be that homophonic model theory in the style presently envisaged delivers its soundness and completeness results in too trivial a way for them to have any significance, but rather than they are not automatically guaranteed to deliver the results in question, contrary to what is said in the final paragraph of the quoted passage. The main point of concern will be raised in Section 4, but immediately we shall be able to see that all is not quite right.

The problem arises in a small (because easily remedied) way already over the presentation of the homophonic model theory under (2) in the passage quoted, and in particular in its treatment of sentence letters (propositional variables). The inductive definition of $I(A)=T$ begins with a condition

$I(p)$ is assigned $T$ or not assigned $T$, for each $p$

which reminds us that the discussion is couched in a potentially non-classical metalogic in which the law of excluded middle is accordingly not to be taken for granted but must be explicitly assumed if it is needed. An incidental correction is called for, in that it is not $I(p)$ that is "assigned" anything but $p$ that is assigned $T$ (or otherwise) by $I$, so we what is really intended is rather

$$
\text { For all sentence letters } p, I(p)=T \text { or } \operatorname{not}(I(p)=T) \text {. }
$$

But was there any need to impose this condition in the first place, supposing that we - or the classically educated among us - have managed to get our

\footnotetext{
${ }^{8}$ In another frequently cited passage from the earlier (Priest 1984, 161), Priest urged a similar line not just for the logic/metalogic distinction but for the language/metalanguage distinction: "The beauty of the paraconsistent approach to logic is that it finally renders the object-language/meta-language distinction unnecessary in any shape or form." The frequent appeals to that distinction in our discussion might seem to make it something Priest would object to, though as long as he is prepared to distinguish between using and mentioning an expression, the hope would be that only minor reformulations here and there would be needed to render the discussion unobjectionable. Some of the discussion of Priest's work in (Humberstone forthcoming) suffers from a failure to distinguish, as is done here, between isological semantics in general and homophonic semantic in particular, as Priest notes at the end of his reply to that contribution in the volume in which (Humberstone forthcoming) appears.
}

Organon F 26 (3) 2019: 340-404 
heads around seeing it as a non-vacuous? Surely not, if this is supposed to be a general treatment, since this gives an unwanted illustration of how the semantics described delivers its verdicts of validity or invalidity, in the present case of the former verdict:

Example 1.2 Let $I$ be an arbitrary interpretation for the language with connectives as described in the passage quoted from Brady and Rush and let us consider for an arbitrary interpretation $I$ and sentence letter $p$, whether or not $I(p \vee \sim p)=T$. The inductive clause for $\vee$ tells us that this is the case iff $I(p)=T$ or $I(\sim p)=T$, and the clause for $\sim$ tell us the second disjunct here holds iff $\operatorname{not}(I(p)=T)$, so presumably altogether the account tells us - and if it doesn't, it doesn't allow us to compute $I(A)$ from the various $I(B)$ for proper subformulas $B$ of $A$ in any instance - that $I(p)=T$ or $\operatorname{not}(I(p)=T)$, which is what $(*)$ says must be satisfied for an arbitrary $I$ and $p$; thus what we have shown is that the formula $p \vee \sim p$ is valid now for any sentence letter $p$. (Could we say instead that $A \vee \sim A$ is valid for any formula $A$ ? We don't know enough about the meta-logic to whether this general form is available. If it is not, eyebrows will naturally be raised as the object logic's claim to being regarded as a logic at all; see note 30.) A more rigorous version of the reasoning here can be presented using the apparatus introduced in Section 2; in particular, we will attend to the kind of equivalence needed to legitimate the substitutivity or replacement properties invoked here.

Now, Example 1.2 is bad news. Nobody said anything about the logics under discussion being restricted to those accepting the law of excluded middle, which is described in (Brady and Rush 2009, 494) as one of the two key properties of classical negation (the other being inference by disjunctive syllogism), and not one to which our authors are especially attracted: "Second, when we come to apply logic to the physical world, we discover that there is no definitive guide enabling the separation of subjects into, for example, those to which the law of excluded middle applies, and those to which it does not." (Brady and Rush 2009, 497). Further clear reluctance to endorse unrestricted appeals to excluded middle can be found in (Brady and Rush 2008). Thus the best thing to do with $(*)$ is to pretend it was never laid down as a condition. ${ }^{9}$

The fact that Brady and Rush did impose that condition should give us pause over the confidence with which the quoted passage finishes:

\footnotetext{
${ }^{9}$ Brady and Rush may have a reply available which hangs on an obscure distinction we shall, in the following section, find them drawing between the interpretational metalogic and the underlying metalogic.
} 
The Soundness and Completeness Theorems are then trivial. For completeness, for a nontheorem $N, I(N)=T$ would fail as a result of the failure of the corresponding metalogical law, applied to totally unconstrained assignments to its variables.

No argument is given in connection with soundness - though the sentiment expressed seems correct and will be illustrated in Section 2 - and the treatment of completeness begins with the same disregard of free variables as we saw in the definition of validity, leaving "I" unbound in the assertion that for an arbitrary nontheorem $N, I(N)=T$ would fail: what $I$ ? No $I$ has been mentioned! Because the definition of validity began with a universal quantifier, what we have to show is that it is not the case that for all $I, I(N)=T$. If, by contrast with intuitionistic logic say, our logic happens to treat $\neg \forall I$ and $\exists I \neg$ as equivalent, then we can formulate the task of showing completeness as that of showing the existence of some interpretation $I$ for which we do not have $I(N)=T$, and in view of Example $1.2 p \vee \sim p$ for a logic lacking this as a theorem (with $p$ a sentence letter), this cannot be done - we precisely do not have all the valid formulas provable. The question of whether, with $\left(^{*}\right)$ discarded, there remain problems about completeness, will remain in abeyance until Section 4, where grounds for a negative response will be given - unless the question is to be reconstrued entirely (a possibility aired in Section 5). The suggestion that we know that not every interpretation verifies $N$ "as a result of the failure of the corresponding metalogical law, applied to totally unconstrained assignments to its variables" sounds like a gesture in the direction of an argument, but can it be spelled out more precisely? We return to this question in the discussion following Example 2.1 below (as well as in Section 5). An incidental issue arising at this point discussed in the Appendix in the longer note 'Soundness without reference to a particular axiomatization'.

\section{More detail}

We may summarize the homophonic model-theoretic treatment of connectives by describing its clauses in the truth-definition schematically. With the " $I(A)=T$ " form replaced by " $I \models A$ ", the general case, for $n$-ary connective \# whose metalinguistic analogue we denote by \#, would be as follows, understood as holding for all $A_{1}, \ldots A_{n}$ :

Organon F 26 (3) 2019: 340-404 


$$
I \models \#\left(A_{1}, \ldots, A_{n}\right) \text { iff \# }\left(I \models A_{1}, \ldots, I \models A_{n}\right) .
$$

Under (2) in the quotation from (Brady and Rush 2009) given above, "not", "\&", " $\vee$ " and "if ...then " are taken as ways of writing what in the notation deployed in (\#) would be written as $\simeq, \underline{\mathbb{L}}, \underline{\vee}, \vec{\longrightarrow}{ }^{10}$ Some may prefer to omit the underlining and say we are dealing with the same connective; certainly every logical principle holding for the various \# of the object language must hold in the meta-logic for the corresponding \#, if the discussion under (2) is taken to be an elaboration of an affirmative answer to the question under (1): "Does this mean that it is the same logic as that of the object language?". Taken literally, this affirmative answer means even more, adding the converse demand: every metalinguistic connective must also be the analogue of some object language connective. This raises the question of the status of the "iff" pervading the example given by Brady and Rush, and retained in the formulation of $(\# / \#)$.

One might think that Brady and Rush assumed that "... iff _" was to be understood as "If ... then and if then ...", and thus already available as a derived connective given the "and" and "if/then" (or $\underline{\&}$ and $\rightarrow$ ) appearing on the right-hand sides of the clauses of the inductive definition of $I(A)$ appearing in the quoted passage. But if the example they are describing there, with an unspecified logic governing the connectives involved in those clauses, is meant to be representative, there is the possibility of the object logic providing us without the materials to define such an "iff". In this case two options present themselves: we could either make it clear that the metalanguage may contain such additional logical vocabulary, not present in the object language, or subject the claim that 'trivial' semantics is always available, whether or not it deserves to be called trivial, to the condition that only such logics are under consideration as provide the additional expressive devices needed.

This, however, would not be what Brady and Rush have in mind, though one does have to look around a bit to see this. The discussion in (Brady and Rush 2009) immediately following that quoted in Section 1 is this (p. 505f.):

There seems to be a paradox here, which needs resolution. In order to do this, we need to find a meta-logic which is something

\footnotetext{
${ }^{10}$ We do not underline metalinguistic quantifiers $\forall, \exists$ since for present purposes they have no analogues in the (purely sentential) object language to contrast with.
} 
of a compromise between mimicking the logic of the object language and using the standard classical meta-logic. Perhaps we can answer this question best by using the distinction between interpretational and underlying meta-logic, made by Brady in (Brady 2003, chapter 13), where the interpretational meta-logic interprets the connectives and quantifiers in an appropriately nonclassical way, while the underlying meta-logic interprets the logic of the meta-linguistic infrastructure supporting the interpretation of the connectives and quantifiers.

Since this follows straight on from the passage quoted in Section 1, we might pause to ask what it is from the end of that passage to say that there "seems to be a paradox here, in need of resolution." The point is presumably that it seems wrong to impose an alien (e.g., classical) metalogic on the (typically non-classical) object logic under discussion, but if we respond to this by instead using the same object logic at the metalogical level, then this trivializes the discussion by making the provision of soundness and completeness results automatic. Not exactly a paradox, perhaps, but something which if it were a problem, might be worth responding to. In Section 4 we shall see that the would-be problem does not quite arise as Brady and Rush think: such results are not automatically available for homophonic model-theoretic semantics. Where they are forthcoming, however, one may wish to do non-homophonic but still 'isological' model theory (to use a term from note 5). Perhaps that is what Brady's interpretational vs. underling metalogic distinction is meant to amount to. Digging a little further, we shall come around to something we can bring to bear more usefully on the earlier passage, and that concerns the significance of the "iff" that appears in it. The excavation in question is deferred to the Appendix ('Brady on the interpretational meta-logic').

Since the logical devices required for homophonic model theory of the kind described as trivial in (Brady and Rush 2009) include not only a suitable "iff" but also the universal quantification (over interpretations $I$ ) used in the definition of validity, ${ }^{11}$ if we want to retain the restriction to purely sentential languages, aspects of the first option will be needed to deal with that, whether

\footnotetext{
${ }^{11}$ Since conditions of the form (\#) above - or those from Brady and Rush - tacitly involve quantification over all interpretations $I$ and formulas $A_{1}, \ldots, A_{n}$, one might discern a universal quantifier needing to be made explicit here too. However, one could conceivably take these conditions as schemata and avoid the issue. But when it comes the notion of validity, especially as this features in antecedent position in an implication, the universal quantification over interpretations is inescapable, as in saying that if a given formula $A$ is valid, it is provable (a consequence of the claim that object logic is complete).
}

Organon F 26 (3) 2019: 340-404 
or not the same route is taken in the case of "iff". (A formula $A$ is defined to be valid in the quotation above if " $I(A)=T$, for all assignments to its variables," which more explicitly must be take to mean " $I(A)=T$ - or " $I \models A$ ", on the =free notation preferred treatment here - for all interpretations $I$ ".) Whether or not we take "iff" to be \# for some (not necessarily primitive) object language binary connective \#, we should pause for a moment to consider what its logical behaviour needs to be like.

The question of what is required of the logic of "iff" raises the prior question of how to conceive of logics for present purposes. Since the envisaged toil-free semantics lays down conditions governing the behaviour of object language connectives such as $\vee$, saying that $I \models A \vee B$ iff $I \models A$ or $I \models B$ (for all interpretations $I$, and formulas $A, B$ ), the logic had better tell us what, by its lights, follows from such conditions. Accordingly it is natural to take logics to be consequence relations, even though the remarks on soundness and completeness at the end of the quotation from (Brady and Rush 2009) above show that their primary conception of logics appears to be as sets of formulas. However, other parts of (Brady and Rush 2009), such as p.502, to say nothing of such ventures as (Brady 1994) and (Brady 1993), show that even when setting out a logic axiomatically, they have their eye on the consequence relation induced by the axiomatization in more or less the familiar way, ${ }^{12}$ and we shall follow suit here.

Having opted to conceive of logics as consequence relations, we can resume our discussion of the issue of "iff", and suggest that a natural way to resolve the issue is to require that we are dealing with an equivalential consequence relation, which is to say a consequence relation $\vdash$ whose language, $L$, say, provides a set of formulas $E(p, q)$ in two variables which is such that for all $A, B \in L$, we have the following, $E(A, B)$ being the set of formulas resulting from substituting $A$ and $B$ respectively for $p$ and $q$ in the formulas in $E(p, q)$ : $E(A, B), C(A) \vdash C(B)$ for all 1-ary contexts $C(\cdot) \quad$ and $\quad \vdash E(A, A)$

\footnotetext{
${ }^{12}$ The familiar - or simple-minded, as it is called in (Humberstone 2010) - way of defining a consequence relation from a Hilbert system with given axioms and rules is that we take $A$ to be a consequence of a set of formulas if $A$ can be obtained from formulas in the set together with axioms, by applications of the rules. In (Brady and Rush 2009) we are not quite dealing with a standard Hilbert system, however, whence the description as "more or less" familiar, because of the presence, as in numerous other publications of Brady's, of what he calls meta-rules. As suggested in (Humberstone 2008, 442), these play the role of sequent-to-sequent rules in a Gentzen system, and we may define the consequence relation induced by a Hilbert system with meta-rules to be the least consequence relation closed (when considered as a collection of single-succedent sequents) under these rules and such that when $A$ can be derived from the axioms and the formulas in a given set by the (ordinary) rules, $A$ is a consequence of that set.
} 
where, on the left, and the " $C(\cdot)$ " notation is as explained in note 45 (in the Appendix), while, on the right what is meant is that for each formula $D \in E(A, A)$, we have $\vdash D$ (i.e., $\varnothing \vdash D)$. To make life easier, we will take the additional step of assuming that $E(p, q)$ contains a single formula, to be written as $p \Leftrightarrow q$; this is done for expository convenience and is not essential to the points under discussion. While no corresponding such connective is presumed to be part of the object language's vocabulary, it may often be that

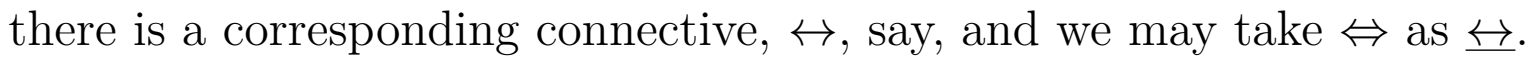

When referring to the consequence relation on the metalanguage for discussing the object-logical consequence relation $\vdash$, we will co-opt the underlining convention just recalled and denote the metalogical consequence relation by $\_$. From a similar desire to make it quite clear whether it is the object language or the metalanguage that is issue, we continue to use capital Latin letters as schematic for object language formulas, but use lower case Greek letters as schematic for formulas of the metalanguage. With all these assumptions and conventions in force the above equivalentiality conditions emerge as (2.1):

$$
\text { (a) } ⺊ \varphi \Leftrightarrow \varphi \quad \text { and } \quad \text { (b) } \varphi \Leftrightarrow \psi, \chi(\varphi)\llcorner\chi(\psi)
$$

understood as holding for all formulas $\varphi, \psi, \chi($ or $\chi(\cdot))$ of the metalanguage, with a similar understanding in the case of the equivalent set of conditions $(2.2)$ :
(a) $\vdash \varphi \Leftrightarrow \varphi$,
(c) $\varphi \Leftrightarrow \psi, \varphi \unrhd \psi$, and
(d) $\varphi \Leftrightarrow \psi \models \chi(\varphi) \Leftrightarrow \chi(\psi)$

The (2.1) formulation has the pleasant feature of echoing for $\Leftrightarrow$ the natural deduction introduction (in the case of (a)) and elimination rules (in the case of (b)) for the identity predicate in first-order logic (a theme taken up in Humberstone 2011, 603f.), while the (2.2) formulation show the relation between having a set (here a singleton set) of equivalence formulas and having a set of implication formulas, as these notions figure in contemporary algebraic semantics: for the latter, retain $(2.2 \mathrm{a}, \mathrm{b})$ and drop $(2.2 \mathrm{c})$, preferably at the same time re-notating " $\Leftrightarrow$ " to " $\Rightarrow$ " to avoid confusion. ${ }^{13}$ Now we can be more

\footnotetext{
${ }^{13}$ Note that these conditions are considerably weaker than those mentioned in the Appendix (note 45) in connection with Rasiowa.
}

Organon F 26 (3) 2019: 340-404 
precise about the "iff" in $(\# / \underline{\#)}$, repeating it here as clarified (though not re-named):

$$
I \models \#\left(A_{1}, \ldots, A_{n}\right) \Leftrightarrow \underline{\#}\left(I \models A_{1}, \ldots, I \models A_{n}\right) .
$$

The point of all this is that to cash out the interpretation-relative truthconditions of complex formulas in terms ultimately of their atomic constituents' truth-conditions in accordance with the homophonic inductive clauses, we need to make replacements in the scopes of various connectives. To take the simplest possible case:

Example 2.1 Suppose, to give the simplest possible illustration, that we have two object-language one-place connectives $\square$ and $\neg$ and accordingly two matching metalinguistic connectives $\square$ and 그, as well $\Leftrightarrow$ as the universal quantification over formulas and interpretations which is invisible in (\#/\#) but taken to be present in the instances of this schema we invoke (with $n=1$ in each case) for these connectives. We want to calculate when $I \models \square \neg p$, say. $(\square / \square)$ tells us that for any $I$ and $A$, we have $I \models \square A \Leftrightarrow \square(I \models A)$, so taking $A$ and $\neg p$ we have

$$
I \models \square \neg p \Leftrightarrow \sqsubseteq I \models \neg p
$$

and similarly invoking $(\square / \square)$ we get

$$
I \models \neg p \Leftrightarrow \neg(I \models p)
$$

Now we may instantiate the (meta-)schema (2.2d) with (2.4) on the left and taking $\chi(\cdot)$ as $\square(\cdot)$ :

$$
[I \models \neg p \Leftrightarrow \neg(I \models p)] \vdash[\sqsubseteq I \models \neg p \Leftrightarrow \unrhd \neg(I \models p)]
$$

Here, to make it clear among a plethora of various turnstiles that the main relational symbol here is the $\underline{-}$, I have put square brackets round the formulas represented as $\varphi \Leftrightarrow \psi$ and $\chi(\varphi) \Leftrightarrow \chi(\psi)$ in (2.2d). Since our semantic theory for the object logic delivers (as (2.4)) the formula on the left, the right-hand side here is a consequence, according to the metalogic $上$, of that theory, and this formula

$$
\square(I \models \neg p) \Leftrightarrow \square \neg(I \models p)
$$

has for its left-hand side the right-hand side of (2.3), and the conditions laid down for $\Leftrightarrow$ suffice to ensure that for any $\alpha, \beta, \gamma$, 


$$
\alpha \Leftrightarrow \beta, \beta \Leftrightarrow \gamma \models \alpha \Leftrightarrow \gamma
$$

so taking $\alpha, \beta$, $\gamma$ as $I \models \square \neg p, \sqsubseteq(I \models \neg p)$, and $\square \neg(I \models p)$ respectively, we get from (2.3) and (2.5), the final stage to be reached in spelling out the truth-on- $I$ conditions of $\square \neg p$ :

$$
I \models \square \neg p \Leftrightarrow \unrhd \neg(I \models p),
$$

which completes our illustration - laborious as it has been for such a simple point - of the reason we need a " $\Leftrightarrow$ " in the metalanguage with the properties we have demanded of it (or at least a set $E(p, q)$ of equivalence formulas to play the $p \Leftrightarrow q$ role).

Let us turn now to the remark with which the passage from (Brady and Rush 2009) quoted in Section 1 concluded:

The Soundness and Completeness Theorems are then trivial. For completeness, for a nontheorem $N, I(N)=T$ would fail as a result of the failure of the corresponding metalogical law, applied to totally unconstrained assignments to its variables.

The sudden reference out of nowhere to $I$ already attracted our attention in Section 1, but here we want to attend to the soundness side of the case. (We return to the issue of semantic completeness in Section 4, and to what Brady and Rush say about it in the passage just quoted, in Section 5.) By way of commentary on the triviality claim for the soundness result, let us take as an example of our object language the purely implicational logic $B C K$. As already noted, what we really need to have in mind is a consequence relation, certainly for meta-logical reasoning and in this case, as already noted, we will need more than just implication in our logical we will also need universal quantification, so if the object logic is given by the consequence relation $\vdash_{B C K}$ defined presently, then the metalogic will be governed by a consequence relation $-上_{B C K}-$ which mimics $\vdash_{B C K}$ propositionally but also includes quantificational and equivalential resources. Section 1 stressed also the need to include an 'iff' surrogate to handle the inductive definition of truth (on an interpretation) and $\vdash_{B C K}$ is well known to provide a set $E(p, q)$ of equivalence formulas in the shape of $\{p \rightarrow q, q \rightarrow p\}$, which, in order to stay as close to the original passage from Brady and Rush, we shall condense into a singleton presently for the case of $上_{B C K}$. Returning to $\vdash_{B C K}$ itself, this consequence 
relation is defined to relates a set $\Gamma$ of formulas to a formula $C$ just when $C$ can be obtained from the formulas in $\Gamma$ by successive applications of Modus Ponens (the rule taking us from $A \rightarrow B$ and $A$ to $B$ ) to formulas which are either elements of $\Gamma$ or instances of the axiom schemes

$$
\begin{aligned}
& (B \rightarrow C) \rightarrow((A \rightarrow B) \rightarrow(A \rightarrow C)) \\
& (A \rightarrow(B \rightarrow C)) \rightarrow(B \rightarrow(A \rightarrow C)) \\
& A \rightarrow(B \rightarrow A)
\end{aligned}
$$

Although in other publications Brady has concerned himself with the semantics of rules and consequence relations, the emphasis in the passage quoted from (Brady and Rush 2009) seems to be on soundness and completeness for logics as sets of formulas and thus as potentially presented by a Hilbert system - skirting around the issue raised in the longer note 'Soundness without reference to a particular axiomatization' on the preceding section (in the Appendix) - which in the current instance amounts to showing the validity ( = truth on all interpretations) of, respectively, all and only those formulas which are $\vdash_{B C K}$-consequences of the empty set.

And it is clear in general outline how the recipe for a soundness proof sketched in the passage quoted is supposed to go. In relatively informal terms, taking the case of $(\mathrm{K})$ as the shortest schema by way of illustration, we need to show that any instance of this schema is valid. So let us take an arbitrary interpretation $I$, with a view to showing that $I \models A \rightarrow(B \rightarrow A), A$ and $B$ as in $(\mathrm{K})$, now taken as standing for particular unspecified formulas rather than as general schematic letters. Let us recall the specific instance of $(\# / \#) \#$ is $\rightarrow$ :

$$
I \models A \rightarrow B \Leftrightarrow(I \models A \rightrightarrows I \models B) .
$$

Giving the name $(\underline{K})$ to the corresponding meta-logical schema, i.e. $\varphi \rightarrow$ $(\psi \rightarrow \varphi)$, we reason as follows, using $\Leftrightarrow$ as above for convenience (though since $\{p \rightarrow q, q \rightarrow p\}$ is a set of equivalence formulas for $\vdash{ }_{B C K}$, we don't actually need to wheel in a new connective here).

$$
\begin{array}{lll}
1 & I \models A \rightarrow(I \models B \rightarrow I \models A) & \text { instance of }(\underline{\mathrm{K}}) \\
2 & I \models A \rightarrow I \models B \rightarrow A & \text { replacing the rhs of } 1 \text { by }(\rightarrow / \rightrightarrows) \\
3 & I \models A \rightarrow(B \rightarrow A) & \text { from } 2 \text { by }(\rightarrow / \rightrightarrows) \text { again }
\end{array}
$$


For line 1, a more explicit annotation might run as follows: for any given formulas $A, B$ of the object language, the metalinguistic formula at line 1 is an instance of the schema $(\underline{K})$. Since 3 is established for arbitrary $I, A, B$ we conclude that every instance of $(\mathrm{K})$ is valid - though we are not formally introducing the metalinguistic universal quantifier into the discussion - and since we can evidently reason similarly in the case of the other axiom schemes, we have all we need by way of a proof of soundness for the axiom system except for showing that the rule Modus Ponens preserves validity. But we can show something stronger: not just that when formulas $A \rightarrow B$ and $A$ are valid (true on all interpretations) so is $B$, but that for each interpretation $I$, if $A \rightarrow B$ and $A$ are true on $I$ then so is $B$. Naturally we need to help ourselves to a little bit of the homophonic semantic theory for $\vdash_{B C K}$, namely $(\rightarrow / \rightrightarrows)$, which can here be understood not as universally quantified but as pertaining to the specific $A, B$ featuring in an arbitrary single application of Modus Ponens. In other words, we can show

$$
[(\rightarrow / \rightrightarrows), I \models A \rightarrow B, I \models A] \models{ }_{B C K}[I \models B]
$$

For this we have worked our way from the three left-hand formula here to that on the right, using the resources of $上_{B C K}$, so, calling our starting formulas 1,2 , and 3 , we may set things out vertically again, writing out the current $(\rightarrow / \rightrightarrows)$ explicitly for line 1 :

$$
\begin{array}{ll}
1 & I \models A \rightarrow B \Leftrightarrow(I \models A \rightarrow I \models B) \\
2 & I \models A \rightarrow B \\
3 & I \models A \\
4 & I \models A \rightarrow I \models B \\
5 & I \models B
\end{array}
$$

From 1, 2 by $(2.2 \mathrm{c})$

From 3, 4 by Modus Ponens for $\rightarrow$

Note that if we use the set $\{p \rightarrow q, q \rightarrow p\}$ of equivalence formulas already mentioned then the appeal to $(2.2 \mathrm{c})$ in line 4 would itself by an appeal to Modus Ponens for $\rightarrow$, as in line 5 .

Accordingly we conclude that whenever $A_{1}, \ldots, A_{n} \vdash{ }_{B C K} B$, for any interpretation $I$, if $I \models A_{1}, \ldots, I \models A_{n}$, then $I \models B$, and not just for the case of $n=0$ : we are not just showing that the outright theorems of $B C K$ are valid, but soundness in the stronger sense that any syntactic consequence by $\vdash_{B C K}$ 
of some formulas is a semantic consequence of them in the sense of being true on every interpretation on which all of them are true. And of course, there is a bit of hand-waving, already acknowledged, on the quantificational front here, but the point is to illustrate that the correspondence between aspects of the meta-logic $\left\llcorner_{B C K}\right.$ and the matching aspects (axioms and rules) of the object logic make the issue of soundness a trivial one. So here I am agreeing with what Brady and Rush don't say but might well have said: we have been discussing soundness, whereas what they actually say concerns a similar match-up between object and meta-logical principles in connection with a toil-free proof of completeness (in the general case). We return to that idea in Section 5. The reason the hand-waving over such things as the quantificational inferences required in the above discussion of soundness, as well as the inductive structure of the proof, is being taken as cause for no great concern is that by the end of the following section we will stop trying to conduct the discussion in a weakening of classical logic and look instead to an extension of classical logic for which, if Brady and Rush's remarks are correct, it should be equally trivial to provide a proof of soundness and completeness w.r.t. homophonic model-theoretic semantics. Since it is the discussion from (Brady and Rush 2009) that we are principally taking up here, a few comparative remarks on related work are postponed to the Appendix ('Bacon and others').

\section{Weaken your metalogic: easier said than done}

So far we have mostly gone along with the idea that it is a simple matter to shift from the use of a classical metalogic for reasoning about a weaker object logic to the use of a weaker - indeed exactly correspondingly weaker - metalogic. It is certainly easier said than done to shift to a weaker or indeed to a stronger metalogic than one finds oneself with, if "shift to" means "come to adopt as one's working logic"; some considerations highlighting the problematic nature of such a procedure can be found in (Berger 2011). ${ }^{14}$ Here

\footnotetext{
${ }^{14}$ The problems seem somewhat exaggerated, in the case Kripke's envisaging a reluctance to endorse $\forall \mathrm{E}$, or universal instantiation ('UI') as it is called in (Berger 2011) (see esp. p. 183 for this issue of this reluctant reasoner), on the part of someone who is not only reluctant to draw conclusions in accordance with this rule but is instead inclined to infer the negations of the instances in question ('Perverse Instantiation', p. 185). One would surely want to say of that the former reasoner does not understand what is written as "all" (or " $\forall$ ") as
} 
we are concerned not so much with endorsing a weaker logic as with being able to see what follows from what from the perspective of such a logic, when the 'what's that are involved are formulated with an indifference to details which are logically immaterial by the lights of one's customary working logic.

There are evidently many issues in the philosophy of logic involved here and the intention will be to by-pass them by changing examples to that we can examine the fate of Brady and Rush's triviality claim for completeness results w.r.t. homophonic model-theoretic semantics when the logic in question is an extension of classical logic rather than a weaker logic proposed as an alternative to classical logic. But to motivate this change of tack, something needs to be said about how in fact it is anything but a simple straightforward matter to shift - in the sense just clarified - to a weaker logic, whether or not one's starting point happens to be classical.

In fact a little has already been said, in Section 1, with the point that not every weaker-than-classical logic endorses the classical equivalence between negated universal statements and existential statements with negated content. Thus we get the oft-remarked inverse relationship between deductive power and discriminatory power. ${ }^{15}$ In the $\neg \forall I$ vs. $\exists I \neg$ case just recalled, for instance, one could define validity ${ }_{1}$ and the validity ${ }_{2}$ of a formula as its truth on all interpretations for the former and as the non-existence of an interpretation which it is false in the latter. These formulations are meant to invoke the $\forall I$ and $\neg \exists I \neg$ regimentations, understood intuitionistically. It does not seem obvious to one at home in a logic in which these are equivalent which, out of validity $_{1}$ and validity 2 best represents what had always seemed to be the single undifferentiated notion of validity. In a similar vein, Kreisel, perhaps the first logician to consider the possibility of an intuitionistically acceptable completeness proof for intuitionistic (predicate) logic (in (Kreisel 1962)) by supplying a second-order notion of validity for Heyting's predicate calculus ${ }^{16}$ and then distinguishing completeness, in respect of a formula A, as $\operatorname{Valid}(A) \rightarrow \operatorname{Prov}(A)$ (to use the earlier notation now for Kreisel's concepts) weak completeness in

universal quantification, while the latter, further, understands "All Fs are Gs as" as "No Fs are Gs". (Here we hedge, as in (Berger 2011), over the issue of restricted vs. unrestricted quantifiers.) Cases of disagreement in reasoning according to rules which go beyond those uniquely characterizing the logical constant in question would, however, be more problematic. (See subsection 4.32 of (Humberstone 2011).) The same applies to Lewis Carroll's prototype, of the tortoise reluctant to reason in accordance with Modus Ponens; in this case a loose parallel to Kripke's Perverse Instantiation is provided by Haack's 'Modus Morons' (in (Haack 1976)).

${ }^{15}$ For references, discussion, and some caveats, see (Humberstone 2007a).

${ }^{16}$ (Dummett 1977, §5.6) provides further discussion of this and other approaches.

Organon F 26 (3) 2019: 340-404 
respect of $A$ as $\sim \sim(\operatorname{Valid}(A) \rightarrow \operatorname{Prov}(A))$, lifting this terminology to a logic (in particular to Heyting's) when the corresponding property is possessed in respect of all formulas. (Note incidentally that this has nothing to do with the usual 'derivability' vs. 'outright provability' contrast between strong and weak completeness or between the similarly named properties from (Bacon 2013), touched on in the longer notes on Section 2 in the Appendix, headed 'Bacon and others'.)

What the adherent of a weaker logic regards as the apparatus for making finer discriminations in the subject matter than are available to the adherent of the stronger logic, who is faced instead with a proliferation problem: how on earth to respond the requirement to 'deconflate'. This of course arises not just with characteristically metalogical vocabulary such as valid and complete (as it does in other specific areas: intuitionistically we have non-empty vs. inhabited sets/species, apartness vs. non-identity of real numbers and so on), but to the core logical vocabulary itself. Sticking with the case of intuitionistic logic as seen from a classical perspective, should one represent "Only $F$ s are $G$ s" as " $\forall x(G x \rightarrow F x)$ " or as " $\forall x(\neg F x \rightarrow \neg G x)$ "? Or again " $\neg \exists x(G x \wedge \neg F x)$ ? Even though as candidate representations from the point of view of English syntax, the last two might appeal on the grounds that the negations involved may explain the appearance in only contexts of negative polarity items - "She only ever visits on Fridays" - semantically and logically one is not accustomed to making much of a fuss about which to write. In fact the first pair here raise another specifically metalogical choice for how to construe completeness for the case in which a contraposed conditional is not equivalent to the original option remaining after a notion of validity has been settled on: do we require that for all formulas $A, \operatorname{Valid}(A) \rightarrow \operatorname{Prov}(A)$, or instead that for all $A, \neg \operatorname{Prov}(A) \rightarrow$ $\neg \operatorname{Valid}(A)$ (with a corresponding distinction for soundness). Although what one usually wants from anything that would be pronounced as "if and only" if would be a connective with the equivalential properties described in Section 2, favouring the choice of $A \leftrightarrow B$ as standardly defined by $(A \rightarrow B) \wedge(B \rightarrow A)$ rather than the intuitionistically non-equivalent $(A \rightarrow B) \wedge(\neg A \rightarrow \neg B)$, which does not support replacement in the same way, who is to say for which occurrences in one's own work a material biconditional should give way to the latter biconditional instead - not to mention other classically equivalent but intuitionistically non-equivalent candidates $-(A \wedge B) \vee(\neg A \wedge \neg B)$, and so on? A further example of this phenomenon is given in the Appendix (see 'Bell and others'). 
An embarras de richesses arises at another level also, when, given a theory with classical logic as its background logic, an attempt is made to specify a corresponding theory with a weaker logic in the background. Thus, for example, (Kreisel 1958), p. 371f. writes "The theory is an extension of Heyting's arithmetic, by which we mean the system obtained by adding the non-logical axioms of classical arithmetic to Heyting's predicate calculus." Here we are not making the familiar point that citing a theory does not suffice to single out any particular axiomatization of it. What is currently problematic, rather, arises from citing a particular axiomatization - and in the context of Kreisel's discussion, the Dedekind-Peano axioms are no doubt what is intended - and then saying that one uses 'these very axioms' with a weaker background logic (here intuitionistic predicate logic) to axiomatize the corresponding weaker theory. And the problem is that although we are talking about the specifically non-logical axioms, they are shot through with logical vocabulary, and different theorists may have made different choices as their official account of the latter vocabulary, differences which are completely immaterial with classical logic in the background, for example regarding $A \rightarrow B$ as abbreviating $\neg A \vee B$, or as abbreviating $\neg(A \wedge \neg B)$, or as primitive - in the last case perhaps alongside $\neg$ with $\wedge$ and $\vee$ takes as defined in any convenient way - but which of these is taken over into the (for the present example) intuitionistic case makes a great difference in view of their non-equivalence according to the weaker logic. That is, there is really no such thing, from the perspective of the weaker logic, as the non-logical axioms to be used for the weaker theory. To illustrate the point with a weakening of classical logic closer to Brady's heart (though far too slight a weakening for his own tastes), we might recall the issues arising (see (Anderson, Belnap and Dunn 1992, 430)) over whether to formulate an induction schema in relevant arithmetic (with underlying logic R) as

$$
\left(A[0] \wedge \forall x\left(A[x] \rightarrow A\left[x^{\prime}\right]\right)\right) \rightarrow \forall x A[x],
$$

where " $x$ " is for the successor of $x$, on the one hand or instead as

$$
A[0] \rightarrow\left(\forall x\left(A[x] \rightarrow A\left[x^{\prime}\right]\right) \rightarrow \forall x A[x]\right),
$$

on the other. A related contrast (conjunctive antecedent vs. iterated implication) has been discussed in the literature on the identity relation in relevant logic: which is the appropriate way of expressing the transition from an identity statement $t=u$ along with a statement involving $t$ to the corresponding conclusion with $u$ ? (See (Mares 1992), (Kremer 1999).) 
To avoid all such challenges, let us switch to a setting in which as much of classical logic is available in the metatheory. Indeed, one of the author's students (Joel Towell) asked the following question on first reading Section 5 of (Brady and Rush 2009): What would they say about what they present as trivial 'toil-less' semantics if it were presented in this style for classical propositional logic itself? A good question: indeed, what difference is there between perfectly orthodox presentation of the semantics - except perhaps that interpretations might be called (Boolean) valuations - and the much-maligned trivial semantics? Soundness and completeness of a particular proof system w.r.t. this semantics does not seem trivial in the sense of being completely uninformative. But the classical switch I have in mind is not quite this because the aim is not so much to rebut the suggestion that homophonic model theoretic semantics is pointlessly uninformative as the suggestion that it is automatically available, with soundness and completeness results effortlessly forthcoming. For this purpose, we need an extension of classical propositional logic, which in view of its Post completeness, will have to be couched in an expansion of its language. The simplest such expansion is the language of monomodal logic, so let us go there now, and show the trouble created for 'semantics without toil' by a phenomenon that is particularly well known in that arena: Halldén incompleteness. (Despite the name, and like Post completeness and incompleteness, Halldén completeness and incompleteness are syntactical notions, their semantic repercussions notwithstanding - though the repercussions envisaged are very different for Kripke semantics than they are for the kind of semantic interpretation Halldén has in mind: see Section $\left.6 .{ }^{17}\right)$

\footnotetext{
${ }^{17}$ (Prior 1957, 54), referring to his proposed modal logic Q, writes "Dr. Alan Ross Anderson has pointed out to me, however, that $\mathrm{Q}$ can be shown to be 'semantically complete' in the sense of S. Halldén, i.e., it contains no theses of the form $A \alpha \beta$ in which $\alpha$ and $\beta$ have no variables in common and yet neither is a thesis on its own." (Here Prior is writing $A \alpha \beta$ for $\alpha \vee \beta$.) This "i.e." is quite misleading. The syntactic property alluded to here is not what Halldén calls semantic completeness, the latter signifying for him (as usual) a link between provability and (a suitable notion of) validity, which Halldén argues cannot be forged when there are provable variable-disjoint disjunctions neither disjunct of which is provable. We will look at this argument in its original setting in the Postscript below (Section 6), with Section 4 concentrating on its bearing on semantic completeness à la Brady and Rush.
} 


\section{Halldén completeness and semantic completeness}

Proceeding with the project just outlined, let us begin by recalling the most widespread contemporary understanding of Halldén completeness for propositional logic. It applies to logics as sets of formulas - historically, originally to modal logics (Halldén 1951) - and if we are thinking of logics as consequence relations, its application depends only on the consequences of the empty set; it presumes the presence of a disjunction connective with more or less the expected properties. In terms of consequence relations those properties would amount to a disjunction $A \vee B$ having for is consequences those formulas which are both consequences of $A$ and consequences of $B .{ }^{18}$ However, as just remarked, its application to a consequence relation depends only on the consequences of $\varnothing$, so we are in effect thinking of logics as sets of formulas and the elements of such set will be described as provable in the logic.

Definition 4.1 A logic is Halldén complete iff whenever $A \vee B$ is provable, and there are no sentence letters appearing in both $A$ and $B$, then either $A$ or $B$ is provable.

Remark 4.2 Halldén's original definition (in (Halldén 1951)) was a little different, having the additional requirement that each of $A, B$ was constructed using a single propositional variable, but this is no longer regarded as a useful demand to build into the definition, though sometimes it is built in with the aid of a qualification: strongly Halldén complete. (See (Schumm 1993a), appearing also as the appendix to (Schumm 1993b), and references there for further discussion.)

Halldén's idea was that for a plausible notion of validity to coincide with provability in a logic, the logic would have to be Halldén complete, and though his argumentation in (Halldén 1951) seems somewhat mysterious today: further detail on this front is postponed to Section 6, for after we have seen the bearing of these issues on Brady and Rush's discussion. Halldén completeness has been discussed in connection with classical propositional logic, numerous

\footnotetext{
${ }^{18}$ The usual understanding of $\vdash$ 's "having disjunction" would be stronger: that $\Gamma, A \vee B \vdash C$ iff $\Gamma, A \vdash C$ and $\Gamma, B \vdash C$, but for present purposes we do not need to require this for $\Gamma \neq \varnothing$.
}

Organon F 26 (3) 2019: 340-404 
intermediate logics - though not for those which, like intuitionistic propositional logic itself, have the disjunction property (understood as per Definition 4.1 except without the condition that $A, B$ have no common sentence letters - making Halldén completeness an immediate consequence of this property), modal logics, ${ }^{19}$ relevant logics (Routley and Meyer 1972, 71), and modal relevant logics (Mares 2003), among others. Here our focus is on (classically based) modal logics, as Halldén's was in (Halldén 1951). The parenthetical "classically based" here reflects the fact that the metalanguage to be used for reasoning about such propositional logics in homophonic model-theoretic semantics will freely avail itself of the deductive resources of classical predicate logic, side-stepping the difficulties noted in Section 3. And in fact this reasoning will be rather familiar ${ }^{20}$ from the case of showing that the set of classical tautologies, is Halldén complete: if neither $A$ nor $B$ is tautologous, so there are Boolean valuations $v, v^{\prime}$ assigning the value $F$ to $A, B$ respectively, then if $A, B$ have no sentence letter in common we can 'combine' $v$ and $v^{\prime}$ into a single Boolean valuation $v^{\prime \prime}$ matching $v$ 's assignments of $T, F$ to the sentence letters in $A$ and matching $v^{\prime}$ s assignments to those in $B$, and we will then have $v^{\prime \prime}(A \vee B)=F$, revealing this disjunction not to be tautologous.

For the case of interest to us here we have interpretations in place of valuations and we have an additional 1-ary connective $\square$ to contend with; this notation is reasonable in view of the traditional very generous definition of what a (mono)modal logic is, though here we extend the idea from logics as sets of formulas to logics as consequence relations. ${ }^{21}$ Concerning $\square$, our semantic account tells us (as in Example 2.1), that for all interpretations $I$ and all formulas $A$ :

19 (Chagrov and Zakharyaschev 1991, 201-206) and (Chagrov and Zakharyaschev 1993, 990-997) provide a wealth of information on Halldén completeness among intermediate as well as modal logics. One historical correction: on p. 201 of (Chagrov and Zakharyaschev 1991) we read that the existence of Halldén incomplete intermediate logics was pointed out in (Halldén 1951). The only intermediate logic in the sense in which Chagrov and Zakharyaschev use the phrase - i.e. for (consistent) superintuitionistic logics - mentioned by Halldén is intuitionistic propositional logic itself, concerning which he makes the point about the disjunction property made in the text here. The authors have perhaps been influenced by the wording of Theorem 2 in (Halldén 1951), with its reference to "S1, S3, or any intermediate calculus," in which it is clear from the context that the last three words mean any logic ('calculus') between S1 and S3.

${ }^{20}$ Given, to cite only discussions already in our bibliography, on the opening page of (Mares 2003), and on p. 862 of (Humberstone 2011).

${ }^{21}$ In this setting we can think of $\vdash$ as a modal logic if $\vdash$ is a substitution-invariant extension in an expansion (by the addition of $\square$ ) of the consequence relation of classical propositional logic, formulated with some functionally complete stock of connectives. 


$$
I \models \square A \Leftrightarrow \square(I \models A)
$$

Here we see " $\Leftrightarrow$ " putting in its regular appearance, so to make life simple we may suppose that the object logic we are currently treating is some Halldén incomplete (normal) extension of S4, in which case we can take $\Leftrightarrow$ as strict equivalence. ${ }^{22}$ For definiteness let us pick the logic mentioned in connection with Schumm in note 22 and give this logic the ad hoc label $\mathbf{S}$ for present purposes, for which purposes nothing about its identity natters beyond its being a normal Halldén incomplete normal extension of S4.

Lemma 4.3 If $p_{1}, \ldots, p_{k}$ are the only sentence letters appearing in the formula $A$ and $I, I^{\prime}$ are interpretations for which we have $I \models p_{i}$ iff $I^{\prime} \models p_{i}$ $(i=1, \ldots, k)$ then $I \models A$ iff $I^{\prime} \models A$.

What follows is a relatively informal proof of Lemma 4.3, though in due course we will write $\Leftrightarrow$ in place of "iff" so as to recall the conditions this connective is presumed to satisfy, according to the current consequence relation $\vdash_{\mathrm{s}}$, taken as the local consequence relation associated with our sample logic $\mathrm{S} .^{23}$ Its metalogical counterpart $上_{\mathrm{S}}$ will have to be a corresponding modal predicate logic, since we need the resources of quantification, and for simplicity let

\footnotetext{
${ }^{22}$ Normal modal logics (as sets of formulas) are sets of formulas in a language with 1-ary $\square$ and some functionally complete set of Boolean connectives, closed under Uniform Substitution, necessitation (prefixing of $\square$ ) and Modus Ponens, and containing all truth-functional tautologies and the formula $\square(p \rightarrow q) \rightarrow(\square p \rightarrow$ $\square q)$; modal logics in general are similarly defined but without the condition concerning this last formula or the condition pertaining to necessitation. The normal extension of a modal logic is the least normal modal logic extending that logic. The supposition that we are dealing with a Halldén incomplete normal extension of S4 presumes that there are such logics, so for definiteness, we may take an example suggested by (Lemmon 1966, 297), namely the normal extension of S4 by: $(p \rightarrow \square \diamond p) \vee \diamond(\square q \vee \square \neg q)$, in which " $\diamond$ " abbreviates " $\square \neg$ ". Lemmon's actual suggestion does not include the word "normal', for which reason a more straightforward example is the extension of S4.4 - of necessity normal (Segerberg 1975) - by the closely related axiom $\square(\diamond p \rightarrow \square \diamond p) \vee \square(\square \diamond q \rightarrow \diamond \square q)$, given in (Schumm 1969) in response to the question of whether there were any modal logics strictly between S4 and S5. (For further information, see Humberstone 2016, 417f.) (Schumm 1993b, 202) notes the existence of uncountably many Halldén incomplete extensions of S4, and following up the reference he gives there shows that we may insert "normal" before "extensions" here. As Lemmon also mentions, J. C. C. McKinsey had earlier observed that there are no Halldén incomplete extensions of S5, and indeed had already isolated (McKinsey 1953, 113) a Halldén incomplete logic between S4 and S5. Further pertinent material can be found in (Schumm 1975).

${ }^{23}$ The local consequence relation associated with a modal logic when the latter is construed as a set of formulas is the relation holding between $\Gamma$ and $A$ when $A$ can be obtained from theorems of the logic and formulas in $\Gamma$ by (successive) applications of Modus Ponens. The label is appropriate because semantically this is a matter of truth at a point in a model, by contrast with global consequence, requiring instead only preservation of the property of being true throughout a model, a syntactic gloss on which would have to allow applications not only of Modus Ponens but also of Necessitation.
}

Organon F 26 (3) 2019: 340-404 
us suppose that $\square$ commutes with $\forall$ according to this consequence relation (the Barcan equivalences). Evidently the basis case for a proof by induction on the complexity ( = number of primitive connectives used in the construction of) $A$ is given by the condition on $I, I^{\prime}$, since in this case $A$ is one of the $p_{i}$. So suppose that $A$ is $\#\left(A_{1}, \ldots, A_{n}\right)$ for some $n$-ary connective \#. In fact in the present case we can take $n \leq 2$ since we may suppose that our object language connectives are just, for example $\neg, \rightarrow$ and $\square$, and for illustrative purposes let us look at the $\square$ case, since this is the only novelty not present in the "combine the Boolean valuations" argument recalled above. Thus our inductive hypothesis is that for a formula $A$ and interpretation $I$ :

$$
I \models A \Leftrightarrow I^{\prime} \models A
$$

and we want to conclude that

$$
I \models \square A \Leftrightarrow I^{\prime} \models \square A
$$

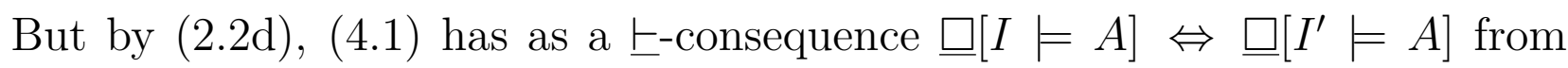
which we get $(4.2)$ via $(\square / \square)$ (and the replacement properties of $\Leftrightarrow$ ).

Remark 4.4 As the above informal argument shows, the wording of Lemma 4.3 is potentially misleading, especially in view of the usual expectations one might have on seeing the abbreviation "iff" - in particular as suggesting that we have here a material biconditional. The point is rather that a suitably generalized version of $(4.2)$ is a $⺊$-consequence, by our metalogical $\_$s4-extending consequence relation $\_$(which in the case of interest below will be $\left\llcorner_{\mathrm{hi}}\right.$ ), of (4.1). It would similarly be potentially confusing to read Lemma 4.3 as saying that whether an interpretation $I$ verifies a formula $A$ is determined by (or depends at most on) whether or not it verifies $p_{i}$ for the various $p_{i}$ occurring in $A$. That might suggest that if $A$ is constructed from one sentence letter $p$ alone, say (for simplicity) $p$, and so as to have a special case of (4.1)-(4.2) before us, that $A$ is $\square p$ and $I \models p$ and as it happens, also $I \models \square A$. Now suppose we have another interpretation $I^{\prime}$ for which, as with $I$ we also have $I^{\prime}=p$. Are we supposing it follows (in the envisaged metalogic) that since $I$ and $I^{\prime}$ thus 'agree' on $p$, each verifying it, that $I$ and $I^{\prime}$ must similarly agree on $\square p$, and thus, since $I \models \square p$, we must have $I^{\prime} \models \square p$ ? That would be disastrous since it would make the context $\square(\cdot)$ - and indeed all contexts left-extensional in the sense of satisfying the condition (here stated for $\square(\cdot)$ ) 
for all formulas $B, C$ and $\vdash$ sound and complete w.r.t the current semantics:

$$
B, C, \square B \vdash \square C
$$

the terminology here being taken from (Humberstone 2011, 454ff.); the same goes for the 'right extensionality' principle (RE) in play in that discussion. This would follow if we had $B, C \vdash B \Leftrightarrow C$, but in general we do not. (Recall we are taking $\vdash$ as the local consequence relation of an associated with on extension of S4 and $\Leftrightarrow$ as strict equivalence. It is because the latter is already in the object language that we have used it in the hypothetical schema $B, C \vdash B \Leftrightarrow C$. What really matters is that we do not have this principle in the metalogic, which would force its validity in the object logic via $(\square / \square)$.) This in the relevant sense of 'agreement' - the sense in which agreement on components yields agreement on compounds - that fact that $I$ and $I^{\prime}$ both verify a formula $A$ both fail to verify $A$ it does not suffice for them to agree on $A$ : for this we require $I \models A \Leftrightarrow I^{\prime} \models B$. Another way - aside form misconstruing the "iff", that is - of falling into this trap would be to adopt the notation warned against in Remark 1.1, and writing " $I(A)=T$ " in place of " $I \models A$ ". Then the initially given details of the case above, namely that $I \models p, I^{\prime} \models p$, and $I \models \square A$, become $I(p)=T, I^{\prime}(p)=T$ and thus that $I(p)=I^{\prime}(p)$, which makes it looks as though $I$ and $I^{\prime}$ cannot but treat $p$ alike, even as it occurs embedded with the context $\square(\cdot)$, and hence that we would then be committed to $I(\square p)=I^{\prime}(\square p)$. To resist such temptations we have Frege's distinction of sense and reference to help, though this is not perhaps a remedy that readily comes to mind in model-theoretic semantics in which possible worlds are not being invoked. (When they are, we have for Frege's distinction, the Carnapian substitute of the intension/extension distinction, but the latter assigns a semantic value as the extension of an expression only relative to a world in a model or interpretation, whereas here we are saying $I(p)=T$ with no such relativization.)

Since there are no constraints on how interpretations treat sentence letters (aside from the ill-fated $(*)$ from Section 1 ), which in the present setting would not count as a constraint anyway, we can re-run the 'combination of valuations' argument for Halldén completeness in the non-modal case, reprised above. Since we are just concerned here what is provable outright according to $\vdash_{\mathrm{s}}$, we refer to the logic as $\mathrm{S}$ :

Organon F 26 (3) 2019: 340-404 
Proposition 4.5 The logic $\mathrm{S}$ is not sound and complete w.r.t. its homophonic model-theoretic semantics.

Proof. Suppose that $\mathrm{S}$ is sound and complete w.r.t. its homophonic modeltheoretic semantics. Take a disjunction $A \vee B$ whose disjuncts share no sentence letters and for which (i) $\vdash_{\mathrm{S}} A \vee B$, (ii) $\nvdash_{\mathrm{S}} A$, and (iii) $\nvdash_{\mathrm{S}} B$ (for example, the $A \vee B$ from Schumm mentioned in note 22). In view of (ii) and (iii) and the supposed completeness of the logic, there are interpretations $I, I^{\prime}$ with $\neg I \models A$ and $\neg I^{\prime} \models B$. Since $A$ and $B$ have no sentence letters in common, we may consider an interpretation $I^{\prime \prime}$ which treats the sentence letters in $A$ as $I$ does, and those in $B$ as $I^{\prime}$ does, in which case $\neg I^{\prime \prime} \models A$ and $\neg I^{\prime \prime} \models B$, and then by Lemma 4.3, $\neg(I \models A \underline{\vee} I \models B)$, so, finally, $\neg I \models A \vee B$, thus contradicting the supposed soundness of the logic, in view of (i).

Fixing on a particular modal logic as $\vdash_{\mathrm{S}}$ was of course just done for definiteness: this argument works for any normal Halldén incomplete extension of S4, and since soundness is not problematic (as Section 2 aimed to show), what is really revealed is a tension between the syntactic property of Halldén incompleteness and the semantic notion of completeness provided by homophonic model theory. One might take, for whatever reason, a dim view, as Halldén clearly did, of Halldén incomplete logics, but that is not really to the point here, which was to query the automatic availability claimed for this semantic approach in the initial quotation from (Brady and Rush 2009). ${ }^{24}$ In Section 6 we look more closely at Halldén's original grounds for dissatisfaction with the logics - especially C. I. Lewis's S1, S2, S3 - displaying this syntactic property: they are strikingly similar for the grounds provided in the proof of Proposition 4.5 for concluding that $\mathrm{S}$ was not amenable to a homophonic semantic treatment, though Halldén's point was couched in different terminology ('normal interpretations': see Section 6), and he may have overestimated Lewis's commitment to such an interpretation in claiming that these weaker logics were semantically intractable by Lewis's own lights.

For the remainder of this section let us attend to a qualm that might be raised about excessive hand-waving in the proof of Proposition 4.5: where precisely do we get the metalogical resources for the crucial $\forall \forall \exists$ step? This

\footnotetext{
${ }^{24} \mathrm{~A}$ referee (or expert reader) points out - something I had completely forgotten - that the present considerations are closely connected with those urged in $\$ 3.3$ of (Williamson 2013) to urge that the set of what are there called metaphysically universal formulas should constitute a Halldén complete modal logic.
} 
claims the existence of a suitable $I^{\prime \prime}$ on the basis of suitably related $I, I^{\prime}$, whereas we have not spelled out explicitly any principles for a formalized metatheory which would secure such existential claims. Rather than doing ourselves to that, however, we shall see, instead, how to sidestep the apparent need to do so by giving a version of the argument which, though it does not apply to the $\mathrm{S}$ under consideration above, readily finds application among the normal (as well as non-normal) modal logics. To move in that direction, we begin by noting a close relative of Halldén incompleteness which has us temporarily jettisoning our recent classical underpinnings.

This close relative, not usually thought of in the same breath as Halldén completeness and incompleteness, is the property of consequence relations called uniformity in (Łoś and Suszko 1958). Not usually - but occasionally: the connection is made explicit by Shoesmith and Smiley in the works cited below, where a uniformity-like condition appears under the name of 'cancellation' which for finitary consequence relations can be taken in the form, with $v b l(\cdot)$ maps a set of formulas to the set of sentence letters occurring in those formulas :

$$
\begin{gathered}
\text { If } \Gamma, \Delta \vdash A \text { and } \operatorname{vbl}(\Gamma \cup\{A\}) \cap \operatorname{vbl}(\Delta)=\varnothing, \text { then } \Gamma \vdash A \text { or else } \Delta \vdash C \\
\text { for all } C .
\end{gathered}
$$

While this condition needs considerable elaboration to cover the case of arbitrary (i.e., not necessarily finitary) consequence relations, it suffices as it stands for present purposes as a necessary condition for a consequence relation to be many-valued in the sense of being determined by a single (logical) matrix. (The elaborations are given in Shoesmith and Smiley 1971 and had already been noted by Ryszard Wójcicki as a necessary supplementation to the treatment in Łoś and Suszko 1958: for the rather complicated details here, see Wójcicki 1974, and p. 278 of Shoesmith and Smiley 1978.)

To see the connection between the claim that a matrix-determined $\vdash$ must satisfy the uniformity/cancellation inset above and the argument that classical propositional logic is Halldén complete, look at what happens when the condition is not satisfied for such a $\vdash$ : the matrix evaluations that end up witnessing the fact that $\Gamma \nvdash A$ and $\Delta \nvdash C$, for some $C$, assign (i) designated values to the formulas in $\Gamma$ but an undesignated value to $A$ and (ii) designated values to the formula in $\Delta$, so since $\Delta$ is variable disjoint from $\Gamma \cup\{A\}$ these assignments can be combined into a single evaluation showing that $\Gamma, \Delta \nvdash A$. (Shoesmith and Smiley 1978, 618f.) go on to explain the 
relationship to Halldén completeness, as standardly understood. (There is no mention of Halldén completeness in the authors' Shoesmith and Smiley 1978.) Kracht $(1999,29)$ boldly redefines Halldén completeness as a property of consequence relations not making any reference to disjunction - though he does mention the usual definition later (p. 141) - but instead using a special case of the inset condition above with $\Gamma=\varnothing$ and $\Delta=\{B\}$, and accordingly announcing (Theorem 1.6.5, p. 30) that all matrix-determined consequence relations are Halldén complete, without the expected further condition that the operation associated with disjunction in the algebra of the matrix in question take any pair of undesignated values to an undesignated value. ${ }^{25}$

Whatever one calls it, this cancellation/uniformity property is enforced, no less than Halldén completeness stricto sensu is, by the homophonic modeltheoretic semantics, via the same "combine the distinctive features of $I$ and $I^{\prime}$ within a single interpretation $I^{\prime \prime}$ " reasoning (even though, yes, we are heading towards a case in which we can by-pass this reasoning). The property in question is famously not possessed by (Johansson style) minimal logic, for whose consequence relation $\vdash_{\mathrm{ML}}$ we have for example $p, \neg p \vdash_{\mathrm{ML}} \neg q$, even though $p, \neg p \nvdash_{\mathrm{ML}} r$ (and thus we do not have $p, \neg p \vdash_{\mathrm{ML}} C$ for all $C$ ) and $\nvdash_{\mathrm{ML}} \neg q$. The violation is even more dramatic if we take a formulation with $\perp$ as primitive (in terms of which $\neg A$ would be defined as $A \rightarrow \perp$ ), but about which $\vdash_{\mathrm{ML}}$ says nothing it does not say about all formulas. Then there are no shared sentence letters on the left and the right in the claim that $\vdash_{\mathrm{ML}} \perp$ since there are no sentence letters at all, yet we have neither $\vdash_{\mathrm{ML}} \perp$ nor: for all $C$, $\perp \vdash_{\mathrm{ML}} C$. In this case, much discussed in (Shoesmith and Smiley 1978), no determining matrix is available because making the value corresponding to the nullary connective $\perp$ designated would conflict with the former and making it undesignated would conflict with the latter. The appropriate incarnation of the general (\#/\#) form for the case of $\#$ as $\perp$ in homophonic model theory

\footnotetext{
${ }^{25}$ This condition was part of the definition of a normal matrix used in (Kripke 1965), in which one reads, concerning the property of Halldén incompleteness, that "Halldén essentially showed that no system with his property can possess a normal characteristic matrix." (Kripke refers the reader to Church's definition of normal matrices or, as Church calls them - following Carnap - normal interpretations: (Church 1956, 117); Halldén has his own definition of 'normal interpretation', quoted in Section 6 below. Normality of matrices in this sense has nothing to do with normality of modal logics, whether in Kripke's originally defined sense requiring the provability of all formulas of the form $\square A \rightarrow A$ - or in the subsequently adopted sense explained in note 22 , which omitted this requirement. The former pertains to the behaviour of the non-modal vocabulary and the latter - essentially - to that of the modal vocabulary.) Note that the algebras used to define the logics on which (Bacon 2013) focuses do not satisfy the condition: the top (and sole designated) element 1 is typically a lattice join of elements distinct from 1.
} 
would then be:

$$
[I \models \perp] \Leftrightarrow \underline{\perp}
$$

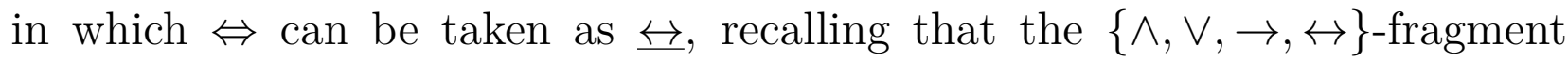
of minimal logic coincides with that of intuitionistic logic. At this point we observe that " $I$ " does not appear free on the right of $(\perp / \perp)$, so we have the consequence that for all interpretations $I, I^{\prime}$ :

$$
[I \models \perp] \Leftrightarrow\left[I^{\prime} \models \perp\right]
$$

from which we might be tempted to conclude that either all interpretations verify $\perp$, in which case we should have $\vdash_{\mathrm{ML}} \perp$, or none do, in which case we should have $\perp \vdash_{\mathrm{ML}} C$ for all $C$, so since neither of these alternatives obtains, minimal logic presents another example in which we do not have soundness and completeness w.r.t. the homophonic model-theoretic semantics. But this would be to forget that the metalogical reasoning is supposed to be as licensed by, for the present case, $\_\mathrm{ML}$, and this inference from (4.3) to a disjunction of universal claims is not thus licensed. This was exactly the kind of nuisance stressed in the previous section occasioned by working not only on but in a logic weaker than one is accustomed to, prompting us to consider classically based ventures.

Undeterred, however, we proceed as with Halldén completeness proper, and shift to a classically based modal logic, so that in the metalogic all of classical predicate logic is available for exploitation. ${ }^{26}$ More specifically, let us pass to the smallest normal modal consequence relation $\vdash_{K}$ (the local consequence relation, in the sense of note 23 , associated with the smallest normal modal logic, $\mathrm{K}^{27}$ ), since in this logic the 'pure' ('letterless') formula $\square \perp$ enjoys the same status - neither provable nor refutable - as $\perp$ itself does in minimal logic. ${ }^{28}$ There is a slight complication in that there is no endogenous $\Leftrightarrow$, so $上 \mathrm{k}$ will have to take this on as a new connective, $p \Leftrightarrow q$ being given the powers it would have had it been defined infinitarily as the conjunction of all

\footnotetext{
${ }^{26}$ Considered as a consequence relation this means that require not only that we have an extension (in a monomodal language) of the consequence relation of classical predicate logic but that it should satisfy also the usual conditional classicality conditions on consequence relations - such as $\rightarrow$-classicality and $\vee$-classicality as they are called in (Humberstone 2011).

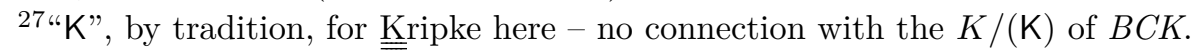

${ }^{28}$ In the terminology of (Kracht 1999, 88), $\square \perp$ is a non-trivial constant (of K); see also the top of p. 142 there, down to and including Proposition 3.7.7 (something mention by name as the online version of the book is differently paginated).
}

Organon F 26 (3) 2019: 340-404 
the $\square^{n}(p \leftrightarrow q)(n \geq 0)$. Invoking both $(\perp / \perp)$ and $(\square / \square)$, we get

$$
[I \models \square \perp] \Leftrightarrow \square \perp
$$

Corresponding to (4.3) above, and for the same reasons, we have (for all $I, I^{\prime}$ ):

$$
[I \models \square \perp] \Leftrightarrow\left[I^{\prime} \models \square \perp\right]
$$

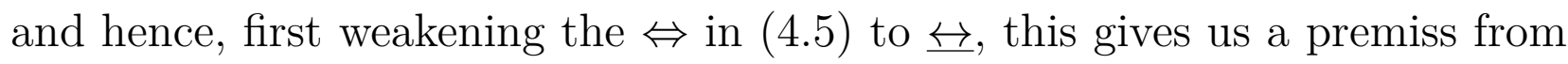
which we may conclude:

$$
\forall I(I \models \square \perp) \underline{\vee} \forall I(\neg I \models \square \perp),
$$

since our reasoning is now entirely classical. For the record then, we have, written in the same style as Prop. 4.5:

Proposition 4.6 The logic $\mathrm{K}$ is not sound and complete w.r.t. its homophonic model-theoretic semantics.

Proof. According to (4.6), one or other disjunct of the object language disjunction $\square \perp \vee \neg \square \perp$ is valid, but since neither is provable in $K$, we have a failure of semantic completeness.

The main point of interest here, as foreshadowed earlier, is that we did not need - as we did for the proof pf Proposition 4.5 - the step that combined one $I$ with a second $I^{\prime}$, occasioned by the invalidity of the disjuncts, into a single $I^{\prime \prime}$, because the r.h.s. of (4.4) does not contain " $I$ ", making it what it says about its l.h.s. independent of any particular interpretation (4.5). Note that in moving from minimal logic into classically based modal logic to illustrate problems coming from the uniformity/cancellation effects of homophonic model theory we have got enough classicality of board for Kracht's redefined version of Halldén completeness, a special case of uniformity/cancellation, to coincide with Halldén completeness. In the present instance we have the 'Halldén unreasonable' disjunction $\square \perp \vee \neg \square \perp$ K-provable without either disjunct's being provable, despite their not sharing a sentence letter. ${ }^{29}$

Digression. Although our concern here is with propositional logics (as object logics) since we are following the lead of Brady and Rush, and it is not

\footnotetext{
${ }^{29}$ If you want to see some sentence letters instead of " $\perp$ " and make this same point, then take distinct letters, $p$ and $q$, and replace the first " $\perp$ " with " $p \wedge \neg p$ " and the second with " $q \wedge \neg q$ ".
} 
quite clear what they would have in mind as a similarly toil-free treatment in the case of predicate logics, it is perhaps worth recalling that (for convenience again) classical predicate logic with identity provides us with sentences constructed (like $\square \perp$ in the example just considered) without the aid of nonlogical vocabulary, such as $\exists x \exists y(x \neq y)$ - call this " $\forall \neq$ " - which the logic does not decide (does not prove or refute) and thus with $\not \models \vee \neg \not \models$ we have a Halldén unreasonable disjunction one of whose disjuncts must be true on all interpretations despite its unprovability - at least if the treatment of predicate logic is close to that of propositional logic. To increase the closeness, we could remove "=" from the language, and even the quantifiers as well, and take $\not \models$ as primitive, as the sole frill added to zero-order logic, this sentential constant being interpreted in conventional model-theoretic terms as true in structures with a universe of two or more elements. (Suitable conditions on $\vdash$ are then given by the unconditional requirement $(i): A(t), \neg A(u) \vdash \not \models$, where $A(t)$ and $A(u)$ are any formulas differing in that one has $t$ at one or more positions in which the other has $u$, and the conditional requirement $(i i)$ : if $\Gamma, F t, \neg F u \vdash C$, then $\Gamma, \not \models \vdash C$, where the monadic predicate letter $F$ and the terms $t, u$ do not occur in the formulas of $\Gamma \cup\{C\}$.) End of Digression.

Returning to propositional logics themselves, let us note that Proposition 4.6 is just one application of something more general. Obviously we get a similar result for $\mathrm{K} 4, \mathrm{~K} 5$, and in general for all the normal modal logics that do not extend KD or KVer, where the latter is the least (normal) modal logic containing $\square \perp$, since among normal modal logics a logic decides all pure formulas (i.e., proves or refutes all formulas constructed without sentence letters) if and only if it proves $\diamond T$ or its negation. But this consideration, and a proof as for Proposition 4.6 above, extend outside of the sphere of normal modal logics, as we may record here:

Proposition 4.7 No modal logic which fails to decide all pure formulas is sound and complete w.r.t. its homophonic model-theoretic semantics.

Proof. Suppose we have a modal logic which does not decide the pure formula A. Then reasoning as in the proof of Prop. 4.6 but with (4.7) in place of (4.6):

$$
\forall I(I \models A) \underline{\vee} \forall I(\neg I \models A),
$$

we have one or other of $\neg A$ valid but ex hypothesi not provable in the logic in question. 
Remark 4.8 As it bears on non-normal modal logics which are not extensions of the smallest congruential modal logic, Proposition 4.7 is sensitive to the choice of the underlying non-modal logic, since if there are no nullary connectives $(T, \perp)$ in that language there are no pure formulas available and the equivalences exploited in note 29 is not available to simulate such formulas. As (Makinson 1973) points out, the smallest modal logic in the language with no pure formulas is Halldén complete, while the smallest such logic in the language providing such formulas is Halldén incomplete. (This gloss on (Makinson 1973) makes use of the characterization given in Proposition 6.7( of the 'congruential' terminology from (Makinson 1971) to apply to modal logics - as sets of formulas - containing $\square A \leftrightarrow \square B$ whenever they contain $A \leftrightarrow B$.)

We mention one interesting application of Proposition 4.7 among the nonnormal modal logics for which the issue raised in Remark 4.8 does not arise:

Example 4.9 (Irvine 2013) extols the virtues of S7 with part of the informal motivation coming from a distaste for Halldén incompleteness, the Abstract heading the paper beginning:

Following Halldén, we define S7 as the system generated by the addition of $\diamond \diamond p$ to S3. Initial motivation for the extension comes from Halldén's paradox. In addition to resolving the paradox, the resulting system generates a helpful framework for comparing classical propositional logic [with various alternative (non-modal) logics].

Similarly on p. 525 we read:

Initial motivation for systems such as S7 thus lay in the fact that S1-S3 have a certain kind of incompleteness, namely that they contain theorems of the form $\alpha \vee \beta$, such that $\alpha$ and $\beta$ have no propositional variable in common and yet neither $\alpha$ nor $\beta$ is a theorem.

And on the following page, noting that one of the disjuncts of some familiar disjunctions used to illustrate Halldén incompleteness extends S3 to S4 and the other to S7 (cf. Proposition 6.7(iii) in our Postscript), Irvine writes:

The resulting systems are, respectively, S4 and S7. In either case Halldén's paradox will be resolved. 
The talk of paradox in this connection derives from the discussion in (Hughes and Cresswell 1968, 268ff.) where the existence of Halldén incomplete modal logics is described as something that "would initially seem strange" and then as seeming "paradoxical at the very least" (both on p. 268), and then (p. 269) they describe Halldén complete logics as those for which the paradox will not arise. It is clear from Hughes and Cresswell's overall discussion, however, they do not wish to commit themselves to finding Halldén incompleteness paradoxical, but rather as reflecting neutrality as between two ways of settling a question: which disjunct to endorse (of a disjunction witnessing Halldén incompleteness). See again the Postscript to the present paper. Irvine appears to be simply assuming that because for the Halldén incomplete S3 we have S3 $=\mathrm{S} 4 \cap \mathrm{S} 7, \mathrm{~S} 4$ and S7 are themselves bound to be Halldén complete. While this was long known to be correct for S4 (Theorem 2 in McKinsey 1953), it was observed not to be the case for S7 in $\$ 4$ of (Shukla 1972) (and, in the same year, in (Pledger 1972), final paragraph), which rather undermines at least this aspect of Irvine's case for S7. Shukla's observation can be put in terms of Proposition 4.7 above by noting that S7 does not decide the pure formula $\square \diamond \diamond \perp$ and is accordingly Halldén incomplete. (Adding that formula as a new axiom gives the logic S8.)

It would be interesting to know in general terms which such logics fall outside of the range for which a homophonic model theory provides a notion of validity (or semantic consequence) matching provability (or syntactic consequence) according to that logic. Do such cases, for instance, simply exhibit Halldén incompleteness or variations on that theme (including here non-uniformity)? ${ }^{30}$ What has been suggested in this section is simply that some logics do lie outside that range, Brady and Rush's claims to the contrary notwithstanding. I say "suggested" rather than "shown" because there may be room for manoeuvre, and some tricky issues arise. Let us have a glance at them.

\footnotetext{
${ }^{30}$ Not that this has anything to do with uniformity, though because of potential confusion here that term is not optimal, let us notice here that often logics are proposed which are not closed under uniform substitution - a fair sample can be found at the top of p. 192 in (Humberstone 2011) - but while these would be obviously outside the range, they are also not in the spirit of Brady and Rush's discussion, whence their exclusion from our concern here, back in note 44 .
}

Organon F 26 (3) 2019: 340-404 


\section{Possible reactions}

Here we consider briefly three possible responses to the findings of the preceding section. A first reaction might be to dismiss Halldén incomplete logics as marginal exceptions to the essentially correct claim of Brady and Rush that the semantical approach they dismiss as trivial because automatically available is indeed automatically available in all but these marginal cases. In reply to this, we recall from the end of Section 4 that the extent of its unavailability has by no means been shown to arise only in the fact of Halldén incompleteness - its extent was left as an open problem - and further, that Halldén incompleteness is by no means to be set aside as a marginal phenomenon. Our postscript (Section 6) aims to substantiate that reply with illustrations of how the phenomenon arises.

A second response which might be anticipated, along the lines of that mentioned in note 9 , is that the discussion above completely ignores the contrast between the interpretational and the underlying metalogic which is stressed in some of the material cited in Section 2 above. Guilty as charged. But even if such a distinction can be made out and shown to salvage the suggestion of triviality or guaranteed success for the position thus bolstered, considerable interest surely still attaches to the homophonic model-theoretic project as discussed here with a straightforward undifferentiated metalogic. If some other authors - Rady and Brush, as it might be - were to make the claim that soundness and completeness were trivially assured w.r.t. such a semantics, it would be worthwhile to explore the areas in which such a claim was an overstatement, and to delimit the precise range of logics - not that any such general result has been offered here - for which the claim would be correct.

A third possible reaction will be described here, on the ultimate success of which no definitive verdict will be offered. The discussion will provide an occasion to revisit the Brady and Rush's claim of triviality for proofs of semantic completeness w.r.t. the homophonic semantics, but it will also lead us into some conceptually challenging terrain. Beginning with the triviality claim, we recall once more that after describing the soundness and completeness theorems (in this setting) as trivial, Brady and Rush say:

For completeness, for a nontheorem $N, I(N)=T$ would fail as a result of the failure of the corresponding metalogical law, applied to totally unconstrained assignments to its variables. 
Contrapositively, if we may permit ourselves such a reformulation (cf. Section 3 ), suppose we are dealing with an unspecified logic, $\vdash$, about which we accordingly do not know whether a formula, say for the sake of example the formula $p \rightarrow((p \rightarrow q) \rightarrow q)$, is provable, but we are told that according to the homophonic model-theoretic semantics this formula is valid. This means that, where $\Sigma$ contains (at least) the set of (universally quantified) (\#/\#) conditions, we have

$$
\Sigma\llcorner\forall I(I \models p \rightrightarrows(I \models p \rightarrow I \models q) \rightarrow I \models q) .
$$

What follows the " $\forall I$ " on the right of the " $\_$" here is indeed an instance of, in Brady and Rush's words "the corresponding metalogical law", namely, in schematic form, $\varphi \rightarrow((\varphi \rightarrow \psi) \rightarrow \psi)$, so the current thought is that Brady and Rush may be taking it our specific instance of this schema could only be a $\underline{\vdash}$-consequence of $\Sigma$ if all instances of this schema were provable in the metalogic. Then, the thought continues, since the metalogic is supposed to coincide, in its propositional fragment, with the object logic, that could only happen if all instances of the corresponding object-linguistic schema were $\vdash$ consequences of $\varnothing$, i.e., if $\vdash p \rightarrow((p \rightarrow q) \rightarrow q)$. Thus we reason from the validity of our formula to its provability. ${ }^{31}$

The reasoning is rather sketchy $-\Sigma$ drops out of the story somewhat mysteriously - but let us suppose that something along these lines is correct. What would an all-gaps-filled explicit version of this argument show? Abbreviating the claim that for all $I, I \models A$ (" $A$ is valid") to " $\operatorname{Val}(A)$ " it begins with the hypothesis that $\Sigma\llcorner\operatorname{Val}(A)$, which asserts the provability of $\operatorname{Val}(A)$ in the metatheory with axioms $\Sigma$ and logic $上$. For brevity we could read this as saying that $A$ is provably valid. So the envisaged perfecting of the argument

\footnotetext{
${ }^{31}$ On the occasion mentioned in the acknowledgments at the end of this paper, Brady confirmed that this was indeed what they had in mind. On the same occasion he made a response distinct from the three canvassed here, namely that mentioned and dismissed immediately after Remark 1.1 above. In a later communication circulated to those who had been in attendance, Brady explained indicated that, on reflection he would like to observe that "the key to toil-less semantics lies in the deductive presentation of semantics, i.e. free semantics (see (Brady 2010)), which was set up in natural deduction, free of disjunctive or existential instantiation. The toil in semantics is in satisfying the requirements of formula induction in standard model-theoretic semantics, e.g. primeness, negation-completeness, existential instantiation. So, our point about the soundness and completeness being toil-less when the object and meta-logics are the same still applies to free semantics. Clearly, in the case of classical logic being used in both object and meta-language, the toil is associated with establishing negation-completeness and the existence of existential witnesses. The same sorts of things would apply for weaker logics, employed in both the object and meta-languages." I include this response here, not because I understand it - or (Brady 2010), for that matter - but for the possible edification of anyone who might.
}

Organon F 26 (3) 2019: 340-404 
sketched above would show that any provably valid formula is provable - where the latter reference is to object-logical provability. (To parallel the semantic characterization, one could equally well say here "is provably provable".) This would be something that could be meant by semantic completeness, and it is not threatened by the Halldén material in Section 4. The argument there was that according to the homophonic semantics we have formulas $A$ and $B$ one of which was true on all interpretations, but whichever it was, it wasn't provable, so there is a failure of semantic completeness. But this is not a failure of semantic completeness in the new sense just isolated. If we imagine the reasoning fully formalized, with $\Sigma$ beefed up (as necessary) so as to support the argument to the existence of a valuation $I^{\prime \prime}$ suitably mimicking $I$ on $A$ and $I^{\prime}$ on $B$, then the best we can hope for is that:

$$
\Sigma 上 \operatorname{Val}(A) \underline{V} \operatorname{Val}(B),
$$

which is to say we do not have $\Sigma \models \operatorname{Val}(A)$ or $\Sigma \vdash \operatorname{Val}(B)$. This means we do not find ourselves with a counterexample to the claim of completeness in the modified sense: we have not given any reason to think that some provably valid formula is unprovable in the object logic. Whether a response along these lines can succeed is unclear. The idea that the modal logic $\mathrm{K}$, for example, is sound and complete w.r.t. a semantic theory that tells us that one of $\square T, \neg \square \top$ is valid but, does tell us which one - that's an idea that would take some getting used to.

\section{Postscript: Halldén completeness, then and now}

This Postscript assumes familiarity with the Kripke semantics for normal modal logics, and in particular with the inductive definition of truth at a point in a model, taken as a triple $\langle W, R, V\rangle$ (on the frame $\langle W, R\rangle$ ). While we begin in Example 6.1 with consequence relations, when discussing modal logics we are thinking of them as sets of formulas; definitions of modal logic and normal modal logic in this setting were provided in note 22. For expository convenience the focus, below, is mainly on normal modal logics. The first time Halldén completeness arose in the literature (Halldén 1951), it was as a feature of non-normal modal logics - in particular, of C. I. Lewis's S1, 
S2, S3 - and there is no substitute, if one wants a clear-headed summary of the early literature on this topic, for pp. 268-272 of (Hughes and Cresswell 1968), perhaps supplemented, for appropriate 'period flavour', by a perusal of Chapter 6 and Appendices II and III of (Lewis and Langford 1959). ${ }^{32} \mathrm{~A}$ particularly attractive feature of their treatment is the idea of a Halldén incomplete logic such as S3 as being "neutral between two conflicting views" (Hughes and Cresswell 1968, 270), in this case those embodied in the two stronger logics S7 (again non-normal) and S4 (normal); the formal underpinning of this feature is given in Proposition 6.7( From this perspective, taken up in (Humberstone 2007b), aspects of which are echoed below, the arguably unfavourable ('something missing') connotations of the 'Halldén incomplete' terminology are misleading: ${ }^{33}$ neutral on this or that issue may be just what one wants to remain. (The issue in (Humberstone 2007b) was realism about non-actual worlds, with an attempt give the 'modal agnosticism' of (Divers 2006) a formal embodiment.) This - perhaps rather superficial - conception of neutrality should not be taken to represent an endorsement of the 'neutral arbiter' status of logical work criticized in a paper mentioned in passing in Section 1 above (as well as in the longer note on that 'On Dummett and others' in the Appendix), (Williamson 2014), in which Williamson warms his readers up with the following (p. 212):

A natural metametaphysical hope is that logic should be able to act as a neutral arbiter of metaphysical disputes, at least as a framework on which all parties can agree for eliciting the consequences of the rival metaphysical theories. An obvious problem for this hope is the proliferation of alternative logics, many of them motivated by metaphysical considerations.

The idea of neutrality as embodied in Halldén incompleteness was not the

\footnotetext{
${ }^{32}$ The same authors' (Hughes and Cresswell 1996), sometimes mistakenly thought to have supplanted the original, contains no discussion of this topic. A later channeling of the less tentative side of Lewis can be found in (Shukla 1972), mentioned in Example 4.9. In Shukla's terminology none of S1, S2, S3, their provenance notwithstanding, counts as a 'Lewis system proper'.

${ }^{33}$ To say nothing about the apparently hostile alternative 'Halldén unreasonable' terminology, used throughout our discussion with no such criticism intended, but simply as a reminder honouring (McKinsey 1953), though here we apply it to the disjunctions witnessing the Halldén incompleteness of a logic rather than, as McKinsey did, to the logics themselves. And, perhaps distancing himself from the suggestion that anything was amiss, recall that McKinsey said "unreasonable in the sense of Halldén" (though "according to" would have been more accurate than "in the sense of"). More on the business of thinking something is wrong with Halldén incompleteness can be found in (Schumm 1993b).
}

Organon F 26 (3) 2019: 340-404 
idea of logic as arbitrating between competing views but rather as a way of refraining from taking sides. The discussion to follow emphasizes themes from (Humberstone 2007b), (Humberstone 2011) - esp. pp.862-872 - and to a lesser extent, (Humberstone 2016) (see the index entries under 'Halldén completeness'). The 'then and now' part of the title is there to record the fact that we will have at least get round to looking at Halldén's own discussion from a contemporary vantage point; the intention to supplement rather than supplant the useful retrospective provided by (Schumm 1993b). But first, an example showing that even for logics, conceived as consequence relations (with disjunction behaving $\vee$-classically), the intersection of two proper extensions does not yield Halldén incompleteness.

Example 6.1 Let us return to the case of minimal logic in and the neutrality of this logic - identified with the consequence relation $\vdash_{\mathrm{ML}}$ of Section 4 - over the interpretation of $\perp$ as a truth or a falsehood. One might, accordingly, think that $\vdash_{\mathrm{ML}}$ is the intersection of two of its proper extensions, one deciding the matter one way, by having a theorem $\perp$ and the other deciding it the other, by having (for all $A) \perp \rightarrow A$ as a theorem. This thought is not quite right, since then the 'Halldén-unreasonable' disjunction $\perp \vee(\perp \rightarrow q)$ would be provable in each of them and yet $\nvdash_{\mathrm{ML}} \perp \vee(\perp \rightarrow q)$ - since $\vdash_{\mathrm{ML}}$ does after all enjoy the disjunction property. In this setting, as one gathers from (Hawranek 1987) (with (Hawranek and Zygmunt 1981) for additional background), ${ }^{34}$ we have to think, instead, in terms of the uniformity or cancellation condition, addressing the consequence relation involved in all its splendour, and not just the consequences of $\varnothing$ according to it. The first thought got one of the two extensions of $\vdash_{\mathrm{ML}}$ right: the smallest extension $\vdash$ of $\vdash_{\mathrm{ML}}$ such that $\vdash \perp$. But the other candidate, it got wrong: what was needed was, rather, the least extension $\vdash$ of $\vdash_{\mathrm{ML}}$ such that for all formulas $C$, we have $\perp \vdash C$. Abbreviating each of Verum and Falsum to its first three letters, let us call these two consequence relations $\vdash_{\mathrm{MLVER}_{\mathrm{V}}}$ and $\vdash_{\mathrm{ML}}$ FAL , respectively. The less obvious part of the claim that $\vdash_{\mathrm{ML}}=\vdash_{\mathrm{ML}} \cap \vdash_{\mathrm{ML}}$ 的 which will be given using classical metalogical reasoning - we make use of the

\footnotetext{
${ }^{34} \mathrm{~A}$ typo in (Hawranek 1987) to watch out for: schema 9 at the base of p. 284 should be $\perp \rightarrow A$ rather than $A \rightarrow \perp$. The explanation is perhaps that one is used to writing the latter as the definition of $\neg A$. The same confusion but in the opposite direction - writing the axiom when attempting to give the definition (in Polish notation this time) - can be found in line 10 of the first page of (Sobociński 1964), when putatively quoting Prior. Less likely to cause confusion: in the middle of p. 287, for "pseudo-relatively lattice" read "relatively pseudo-complemented lattice".
} 
(easily established) lemma that for any $\Gamma \cup\{A\}$ :

$$
\Gamma \vdash_{\mathrm{ML}} A \text { iff } \Gamma, \perp \vdash_{\mathrm{ML}} A .
$$

Now, contrapositively, we need to show that if $\Gamma \nvdash_{\mathrm{ML}} A$ then either $\Gamma \nvdash_{\mathrm{ML}} A$ or $\Gamma \nvdash_{\mathrm{ML}}$ FaL $A$. Invoking the lemma above, this amounts to showing that $\Gamma \nvdash_{\mathrm{ML}} A$ then either $\Gamma, \perp \nvdash_{\mathrm{ML}} A$ or $\Gamma \nvdash_{\mathrm{ML}}$ FL $A$. This we show by assuming (1) $\Gamma \nvdash_{\mathrm{ML}} A$ and (2) $\Gamma, \perp \vdash_{\mathrm{ML}} A$, and inferring from (1) and (2) that $\Gamma \nvdash_{\mathrm{ML}} A$. In view of (1), by a Lindenbaum construction we can extend $\Gamma$ to some $\Gamma^{+}$ with the property that $\Gamma^{+} \nvdash_{\mathrm{ML}} A$ while for any proper superset $\Delta$ of $\Gamma^{+}$, we have $\Delta \vdash_{\mathrm{ML}} A$. In view of $(2), \perp \notin \Gamma^{+}$, which implies that $\Gamma^{+} \nvdash_{\mathrm{ML}_{\mathrm{FAL}}} A$, since $\Gamma^{+}$is accordingly closed under the transition from $\perp$ to arbitrary $C$. Now $\Gamma^{+} \supseteq \Gamma$, so, as we wanted, $\Gamma \nvdash_{\mathrm{ML}_{\mathrm{FAL}}} A$. As (Hawranek 1987) points out, each of (what we are calling) $\vdash_{\mathrm{ML} \text { VER }}$ and $\vdash_{\mathrm{ML}_{\mathrm{FAL}}}$ is determined by a single matrix, in which $\perp$ is assigned a (indeed the) designated element and an undesignated element respectively, meaning that $\vdash_{\mathrm{ML}}$ is determined by a pair of matrices and accordingly ( since it is not determined by any single matrix) has 'degree of complexity' 2. From the perspective of Kripke-style model theory (Segerberg 1968) we can work with pointed frames $\langle U, \leq, Q, u\rangle$ in which $u \in U$ and $Q \subseteq U$ is upward closed under the partial ordering $\leq$ of $U$, an order w.r.t. which $u$ is a least element. In any model on such a frame, $Q$ provides the set of points at which $\perp$ counts as true (and the model provides for each propositional variable a similarly $\leq$-closed truth-set, the connectives $\wedge, \vee, \rightarrow$ being treated as per the Kripke semantics for intuitionistic logic). $A$ is a semantic consequence of $\Gamma$ relative to a class of such frames just in case in any model on one of those frames, if every formula in $\Gamma$ is true at the distinguished point $u$ in the model then so is $A$. Then $\Gamma \vdash_{\mathrm{ML}} A$ just in case $A$ is a semantic consequence of $\Gamma$ relative to the class of all frames, $\Gamma \vdash_{\mathrm{MLVER}_{\mathrm{VER}}} A$ just in case $A$ is a semantic consequence of $\Gamma$ relative to the class of all frames $\langle U, \leq, Q, u\rangle$ in which $u \in Q$, and $\Gamma \vdash_{\mathrm{ML}_{\mathrm{FAL}}} A$ just in case $A$ is a semantic consequence of $\Gamma$ relative to the class of all frames $\langle U, \leq, Q, u\rangle$ in which $u \notin Q$. It is only this last case which is not covered in (Segerberg 1968), where logics are taken as (certain) sets of formulas rather than as consequence relations, and the set of (as we would put it) $\vdash_{\mathrm{ML}}$-consequences of the empty set is referred to as $J$. The closest approximation to the $\vdash_{\mathrm{ML}}$ Fa case covered in (Segerberg 1968) is that of intuitionistic logic, in which the corresponding frame condition is that $Q=\varnothing$, giving us, by contrast with the case of $\vdash_{\mathrm{ML}_{\mathrm{FAL}},}, \rightarrow A$ (for all $A$ ) as a consequence of the empty set rather than just $A$ as a consequence of $\perp$ 
(i.e., of $\{\perp\}$ ). As this underlines for us, $\vdash_{\mathrm{ML}_{\mathrm{FAL}}}$ does not have the 'Deduction Theorem' property. In fact, by strengthening the above condition " $u \notin Q$ " to $Q=U \backslash\{u\}$, we get an extension of Minimal Logic in which if $\neg A$ is defined as $A \rightarrow \perp$ we have $\vdash A \vee \neg A$ for all $A$ : see (Humberstone 2006, 64f.). (In fact $\perp$, so interpreted is written as $\Omega$ and $\neg$ as $\neg \Omega$ so that we can have this new constant alongside a 'nowhere true' constant $\perp$ and an $(A \rightarrow \perp)$-style negation - all of which means we have an extension, in an expanded language, of intuitionistic rather than just minimal logic.)

Example 6.1 invites us to draw a couple of morals. The first is the main intention of the example, namely that the focus on Halldén incompleteness of a logic as a reflection of neutrality between extensions works well in classically based modal logics but is not all-purpose indicator of a logic's being the intersection of two of its proper extensions in this way, since minimal logic is such an intersection but is not Halldén incomplete by the disjunction property argument at the start of Example 6.1. (Similar considerations arise in (Miura 1966).)

Modal logics abound in which provability does indeed coincide with a perfectly respectable notion of validity - validity over this or that class of Kripke frames - despite these logics being Halldén incomplete, which Halldén expected would rule out this possibility. But he was writing in advance of the development of the Kripke semantics and we will revisit his original qualms after talking about, in particular normal modal logics, with that semantics in mind.

Definition 1 of (Halldén 1951, 127) defines a logic, taken as a set of formulas, to be "complete with respect to an interpretation $i$ ", if and only if that set coincides with the set of formulas which "according to $i$, express true propositions", but does not define what an interpretation or a proposition is or what it is to express a proposition according to an interpretation, though from Definition 2, it seems that propositions can be identified either with or via (first-order?) predicate-logical formulas. The interpretations $i$ in play are evidently not to be confused with interpretations $I$ of the kind in play in our discussion of Brady and Rush. In giving Halldén's Definition 2 below, I have replaced his " $(x) \cdot f x$ " notation with " $\forall x(F x)$ " (etc.). This Definition 2 , quoted along with a remark on C. I. Lewis, declares an interpretation $i$ to be a normal interpretation - cf. note 25 - of a logic $C$ (construed a set of formulas), if and only if: 
for all $C$-formulas $P$ and $Q$ which are such, that (1) Each contains only one variable, (2) They have no variable in common, it holds that some monadic functions $F$ and $G$, and some dyadic function $H$ are such that (3) $P$ expresses the proposition $\forall x(F x)$, and $Q$ expresses $\forall y(G y)$, (4) $P \vee Q$ expresses $\forall x \forall y H(F x, G y)$, (5) If $\forall x \forall y(H(F x, G y))$ is true, then either $\forall x(F x)$, or $\forall y(G y)$ is true.

It can immediately be seen that Lewis's own interpretation of the Lewis calculi is a normal interpretation of them. That conditions (3) and (4) are fulfilled is trivial. That (5) is fulfilled partly depends upon the fact that the interpretation assigned to $P \vee Q(\ldots)$ permits the inference from $\forall x \forall y(F x \vee G y)$ to $\forall x(F x) \vee \forall y(G y)$, when $F$ and $G$ contain no free variables.

Now one may well be puzzled by all these universal quantifiers appearing out of thin air, and think vaguely that they are quantifying over points in a Kripke model - but of course such models had no currency when (Halldén 1951) was published. From the point of the Kripke semantics - and indeed from any semantic point of view - the talk of interpreting modal formulas in this way seems to stand in need of a clarification as to whether the interpretation is intended to march in step with the truth of the modal formula or with its validity. From the specifically Kripke-semantic perspective this contrast cross-cuts another of obvious relevance: whether we are speaking locally (truth vs. validity at a point - as explained before Definition 6.2 below or globally (truth throughout a model vs. validity on a frame). At this juncture we can do no better than display Schumm's discussion of the implicit "combination of assignments" here (from (Schumm 1993b, 198)); here $\alpha$ and $\beta$ are the disjuncts of a candidate Halldén-unreasonable disjunction and the square-bracketed insertion is an clarificatory addition:

Now suppose neither $\alpha$ nor $\beta$ is valid, where $\alpha$ and $\beta$ share no variables. This means only that there are worlds (possibly different) in frames (possibly different) at which $\alpha$ and $\beta$ can be made false (possibly under different variable assignments). There is no guarantee, nor any evident reason why there should be, that there will be a single world in a single frame at which $\alpha$ and $\beta$ can both be made false [at the same point] under the same variable assignment, which is what it would take for $\alpha \vee \beta$ to lack validity. 
The reasoning here commented on is a contraposed version of that from the quotation from Halldén, so Schumm is suggesting that what Halldén's universal quantifiers are ranging over are potential assignments to the propositional variables or sentence letters, and what Schumm is emphasizing is a possibility provided by the Kripke semantics and required for the semantic description of modal logics in general - though in elaborating the below, we concentrate on normal modal logics for simplicity - is that these assignments themselves are sets of points (or world) which may differ in their logical properties. To spell out the kind of heterogeneity involved here we need first to define the notion used above, of validity at a point: a formula is valid at a point in a frame when it is true at that point on any model on the frame. ${ }^{35}$ This notion of a valuation $V$ is of the assignment to all the sentence letters at once of subsets of the universe $W$ of a frame $\langle W, R\rangle$ to obtain a model $\langle W, R, V\rangle$ on that frame, whereas the passage from Halldén handles the effects of such a valuation piecemeal, universally quantifying over the assignment to this (propositional) variable and then to that variable,..., and of course he is not specifically thinking of the objects assigned as sets of points in some frame (or model structure, as Kripke used to put it). We can think of the remarks quoted from Schumm above as doing two things. In the first place, it tells us that a more suggestive notation than Halldén's might involve writing $\forall p$ and $\forall q$ rather $\forall x$ and $\forall y$ (though Halldén did not actually use the " $\forall$ " notation) with what look like familiar individual variables. In fact, apparently independently of Halldén, though a few years later, Jerzy Łoś took worked with the corresponding notion in first-order theories - variously called $O$-completeness or quasi-completeness - where it is individual variables that are involved. ${ }^{36}$ The second thing the passage from Schumm does is alert us to the fact that the set of formulas valid at any given point in a Kripke frame is indeed Halldén complete, ${ }^{37}$ and, if we were so inclined, we could then par-

\footnotetext{
${ }^{35}$ We speak of truth when particular valuations - expanding frames to models - are at issue, and of validity when universally quantifying away the reference to such valuations.

${ }^{36}$ See (Łoś 1955), (Łoś and Suszko 1955); Suszko remarks on the relation to Halldén in later publications, such as (Suszko 1971) and (Suszko 1975). One need not consider non-logical theories to find failures of 'quasi-completeness': consider the open formula, free variables having the generality interpretation $\forall y(y=$ $z) \vee \exists z(z \neq u)$ provable in classical predicate logic with identity though neither of the (individual!) variabledisjoint disjuncts is. One could of course insert the invisible universal quantifiers binding " $z$ " and " $u$ " here, but this version looks (slightly) more like the Halldén phenomenon in sentential logics without propositional quantifiers.

${ }^{37}$ This is something to be stressed in any introduction to the topic - as, for example, in Section 4 of (Humberstone 2005).
} 
lay Halldén's dissatisfaction with modal logics lacking that property into a Kripke-semantical setting as an argument that frames in which different formulas are valid at different points have no place in explicating modality. But even if such an argument could be made out, modal logics in the sense currently under discussion have nothing specifically to do with modality - if that means: with necessity and possibility - but are a broad generalization aspiring to encompass many applications (such as the tense logic of 'ending time' to cite a well-known Halldén incomplete case). Even in the case of metaphysical necessity itself, as mentioned in the opening paragraph of this Postscript, a case can be made for Halldén incompleteness on hedging-one's-bets grounds more specifically for a failure of global Halldén completeness as defined below (contrasting in this respect with the tense-logical case just mentioned). This is the line explored in (Humberstone 2007b), for instance.

Definition 6.2 A frame $\mathcal{F}=\langle W, R\rangle$ is weakly homogeneous if there do not exist a pair of formulas $A, B$ for which $W=W_{A} \cup W_{B}$, where $A$ is valid (in $\mathcal{F}$ ) at each point in $W_{A}$, while $B$ is not valid at every such point, and $B$ is valid (in $\mathcal{F}$ ) at each point in $W_{B}$, while $A$ is not valid at every such point.

Remark 6.3 The word "weakly" is included here because a stronger notion of homogeneity could be introduced to describe a frame in which all points validates the same formulas. Stronger still would be the 'language-free' notion of a frame $\langle W, R\rangle$ as homogeneous when for any $x, y \in W$ there is some automorphism of the frame which maps $x$ to $y$. (This last notion, taken from first-order model theory, has some currency in the Halldén completeness literature - for example in (van Benthem and Humberstone 1983) and (Kostrzycka 2015). Incidentally, a variant of Theorem 2 of (van Benthem and Humberstone 1983), tailored to the Routley-Meyer semantics for relevant logic, appears independently as Theorem 5.15 of (Routle, Meyer and Brady 1982).)

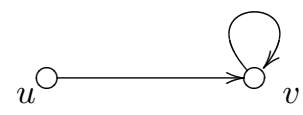

Figure 1: A Weakly Homogeneous Frame

Example 6.4 The frame depicted in Figure 1 is weakly homogeneous despite its points not all validating the same formulas, since $p \rightarrow \square p$ is valid only at 
$v$. (The same goes for $\square p \rightarrow p$.) But the weak homogeneity condition is still satisfied because every formula valid at $u$ (in this frame) is also valid at $v$. (To see this consider the p-morphism mapping each of $u, v$ to the sole element of the one-element reflexive frame.)

Definition 6.5 A class $\mathbb{C}$ of frames is weakly homogeneous if there do not exist a pair of formulas $A, B$ for which $\mathbb{C}=\mathbb{C}_{A} \cup \mathbb{C}_{B}$, where $A$ is valid on each frame in $\mathbb{C}_{A}$ while $B$ is not valid on each such frame, and $B$ is valid on each frame in $\mathbb{C}_{B}$, while $A$ is not valid on each such frame.

Halldén completeness tout court was officially introduced in Definition 4.1; now we introduce for comparative purposes a strengthening of that notion: ${ }^{38}$

Definition 6.6 A modal logic (as a set of formulas) is globally Halldén complete if for any pair $A, B$ of formulas, if $A, B$ share no sentence letters and are such that $\square^{m} A \vee \square^{n} B$ is provable in the logic for all $m, n \geq 0$ (the exponents indicating iteration), then either $A$ or $B$ is provable in the logic.

Let us write $\mathbf{L}(\mathbb{C})$ to denote the modal logic - always a normal modal logic - determined by the class of frames $\mathbb{C}$, abbreviating " $\mathbf{L}(\{\mathcal{F}\})$ " to " $\mathbf{L}(\mathcal{F})$ ". We collect some more or less straightforward consequences of the definitions of the concepts involved in them. Note that a 'modal logic' is here understood in the set-of-formulas sense; part $(i)$ is Corollary 6.41.6 in (Humberstone 2011) and (iii) is a famous observation appearing in both (Kripke 1965) and (Lemmon 1966).

Proposition 6.7 (i) If $\mathcal{F}$ is a weakly homogeneous frame, then $\mathbf{L}(\mathcal{F})$ is Halldén complete.

(ii) If $\mathbb{C}$ is a weakly homogeneous class of frames, then $\mathbf{L}(\mathbb{C})$ is globally Halldén complete.

(iii) A modal logic is Halldén complete iff it is not the intersection of two modal logics properly extending it.

\footnotetext{
${ }^{38}$ As mentioned in note 28 of (Humberstone 2007b), the phrase globally Halldén complete is used in a different sense in (Kracht 1999). Recalling the discussion in Section 4 in which Kracht used the Halldén completeness terminology for a variation on the condition of uniformity (or cancellation) for consequence relations, let us add the further clarification that when this terminology is used sans phrase, it is the local consequence relation associated with a logic that Kracht has in mind - see note 23, and "globally Halldén complete" is used in (Kracht 1999) for the case in which it is, instead, the global consequence relation that is involved.
} 
(iv) A normal modal logic is globally Halldén complete iff it is not the intersection of two normal modal logics properly extending it.

Part (iii) is a famous observation appearing in both (Kripke 1965) and (Lemmon 1966), while part $(i)$ here is Corollary 6.41.6 in (Humberstone 2011), derived as a consequence of the observation that a variable-disjoint disjunction is valid at a point in a frame just in case one of the disjuncts is. Slightly varying Example 4.3 from (Humberstone 2007b) to illustrate some of these concepts, given a frame $\mathcal{F}=\langle W, R\rangle$ call a point $x \in W$ 1-symmetric ("in $\mathcal{F}$ ", though we take this as given by the context in future) just in case for all $y \in W$ if $R x y$ then $R y x$, and 1-transitive just in case for all $y, z \in W$, if $R x y$ and $R y z$ then $R x z{ }^{39}$

Example 6.8 If we are interested in the class of frames in which each point is 1-symmetric or 1-transitive or both), it is easy to see that the modal logic determined by this class of frames is the smallest normal modal logic containing the formula $(p \rightarrow \square \vee p) \vee(\square q \rightarrow \square \square q)$ - evidently a Halldén incomplete logic. On the other hand we may instead take an interest in the logic determined by the class of frames which are symmetric or transitive or both (i.e., in which every point is 1-symmetric or every point is 1-transitive - though here we could equally well replace the point properties with those alluded to in note 39: 2-symmetry on the one hand or 2-transitivity or 3-transitivity on the other). In this case, we find that the logic in question is the smallest normal modal logic containing all formulas

$$
\square^{m}(p \rightarrow \square \diamond p) \vee \square^{n}(\square q \rightarrow \square \square q),
$$

with $m, n \in N a t$, and now our logic is not just Halldén incomplete but globally Halldén incomplete (not globally Halldén complete, that is).

Let us observe in closing that it isn't always necessary to explicitly bolster the disjunction with arbitrarily iterated occurrences of $\square$ in the manner of Example 6.8, though, because we can let the infrastructure of the normal modal logic in play do that work for us. Consider for instance, the logic $\left(\mathrm{KT}_{c}\right.$ in one popular nomenclature due to Chellas and employed in Humberstone 2016): the smallest normal modal logic containing the formula $p \rightarrow \square p$, determined

\footnotetext{
${ }^{39}$ There are also concepts in this vicinity, of 2-symmetry - mentioned in (Humberstone 2007b) - and of 2- and 3-transitivity - see (Humberstone 2016, $185 \mathrm{ff}$.), though to save perusing these sources, let's just say: work your way through the variables used in these first-order conditions.
}

Organon F 26 (3) 2019: 340-404 
by the class of frames in which each point bears the accessibility relation to at most itself. Rewriting this axiom as ( $p \leftrightarrow \square p) \vee \square \perp$ we reveal its (well-known) Halldén incompleteness. We leave the interested reader to check - 'globality for free' - that in fact in this case all the formulas $\square^{m}(p \leftrightarrow \square p) \vee \square^{n} \square \perp$ are already provable in this logic without further ado (without either disjunct being provable). In $\mathrm{KT}_{c}$ the behaviour of $\square$ is exactly what it would be if we were to take $\square A$ as having being added to classical truth-functional logic by defining it as $\Omega \vee A$, where $\Omega$ is a sentential constant about which nothing else need be said. The reason behind this choice of notation is not to remind the reader of (the end of) Example 6.1, but rather to recall the notation of (Porte 1979), in which the necessity operator of Łukasiewicz's Ł-modal logic - not a normal modal logic, shown to be Halldén incomplete in (Anderson 1954) - is observed to be similarly definable using an unspecified constant $\Omega$ but this time with the definiens $\Omega \wedge A .{ }^{40}$ Operators so introduced are evidently in the class of those called extensional (in sentence position) though not truth-functional in (Humberstone 1988), where all such connectives are observed to yield Halldén incompleteness, the disjuncts (of a suitable witness disjunction) running through alternative candidate truth-functions. ${ }^{41}$

\section{Acknowledgments}

For assistance in numerous ways, I thank Andrew Bacon, Max Cresswell, Rohan French, Patrick Girard, David Makinson, Shawn Standefer, the editors of this special issue and the external expert reader they consulted, as well as those in attendance (Graham Priest, in particular) at the Workshop in Honour of Ross Brady organized by Shawn Standefer at the University of Melbourne in August 2018, where this paper was presented.

\footnotetext{
${ }^{40}$ (Sotirov 2008, 317), notes that this observation had appeared ten (or more) years previously in a 1967 paper in German by D. Vakarelov. (Just for the record, the bibliography of (Porte 1979) includes a publicly distributed - though not actually published - conference handout by Porte incorporating this observation and dated 1962. The corresponding possibility operator is accordingly definable by $\Omega \rightarrow A$, something observed in print even earlier, at p. 189 of Prior 1956.) More information on the 'sentential constant' approach to the L-modal system, and on the the connection between this and $\mathrm{KT}_{c}$, can be found in (Font and Hájek 2002), where $\Omega$ is mostly written as $L$.

${ }^{41}$ The terminology used here to describe these cases is not ideal. It is improved in $\S \S 3.1-3.2$ of (Humberstone 2011).
}

Organon F 26 (3) 2019: 340-404 


\section{References}

Aberdein, Andrew and Read, Stephen. 2009. "The Philosophy of Alternative Logics", in The Development of Modern Logic, edited by L. Haaparanta, pp. 613-723. Oxford: Oxford University Press. https://doi.org/10.1093/acprof : oso/9780195137316.003.0041

Anderson, Alan R. 1954. "On the Interpretation of a Modal System of Łukasiewicz", Journal of Computing Systems 1: 209-210.

Anderson, Alan R., Belnap, Nuel D. and Dunn, Jon M. 1992. Entailment: the Logic of Relevance and Necessity, Vol. II, Princeton: Princeton University Press.

Bacon, Andrew. 2013. "Non-classical Metatheory for Non-classical Logics." Journal of Philosophical Logic 42(2): 335-355. https://doi.org/10.1007/s10992-012-9223-9

Bell, John L. 2008. A Primer of Infinitesimal Analysis, Cambridge University Press, Cambridge. (First edition 1998.)

Belnap, Nuel D. and Dunn, Jon M. 1981. "Entailment and the Disjunctive Syllogism.". In Contemporary Philosophy: A New Survey, Vol. 1, edited by G. Fløistad, pp. 337-366. The Hague: Martinus Nijhoff.

van Benthem, Johan and Humberstone, Lloyd. 1983. "Halldén-completeness by Gluing of Kripke Frames." Notre Dame Journal of Formal Logic 24(4): 426-430. https://doi.org/10.1305/ndjfl/1093870446

Berger, Alan. 2011. "Kripke on the Incoherency of Adopting a Logic." In Saul Kripke, edited by A. Berger, pp. 177-207. Cambridge: Cambridge University Press.

Brady, Ross T. 1993."Rules in Relevant Logic - II: Formula representation." Studia Logica 52(4): 565-585. https://doi.org/10.1007/BF01053260

Brady, Ross T. 1994. "Rules in Relevant Logic - I: Semantic Classification." Journal of Philosophical Logic 23(2): 111-137. https://doi.org/10.1007/BF01050340

Brady, Ross T. (ed.) 2003. Relevant Logics and their Rivals, Vol. 2. Aldershot: Ashgate.

Brady, Ross T. 2010. "Free Semantics." Journal of Philosophical Logic 39(5): 511-529. https://doi .org/10.1007/s10992-010-9129-3

Brady, Ross T. and Rush, Penelope. 2008. "What is Wrong with Cantor's Diagonal Argument?" Logique et Analyse 51(202): 185-219.

Brady, Ross T. and Rush, Penelope. 2009. "Four Basic Logical Issues." Review of Symbolic Logic 2(3): 488-508. https://doi.org/10.1017/S1755020309990219

Braüner, Torben. "Modal Logic, Truth, and the Master Modality." Journal of Philosophical Logic 31(4): 359-386. https://doi .org/10.1023/A:1019992820056

Chagrov, Alexander and Zakharyaschev, Michael. 1991. "The Disjunction Property of Intermediate Propositional Logics." Studia Logica 50(2): 189-215. https://doi.org/10.1007/BF00370182

Chagrov, Alexander and Zakharyaschev, Michael. 1993. "The Undecidability of the Disjunction Property of Propositional Logics and Other Related Problems." Journal of Symbolic Logic 58 (1993), 967-1002.

Organon F 26 (3) 2019: 340-404 
Church, Alonzo. 1956. Introduction to Mathematical Logic, Vol I. Princeton: Princeton University Press.

Czelakowski, Janusz. 2001. Protoalgebraic Logics. Dordrecht: Kluwer.

Divers, John. 2006. "Possible-Worlds Semantics Without Possible Worlds: The Agnostic Approach." Mind 115(458): 187-225. https://doi.org/10.1093/mind/fzl187

Dummett, Michael. "Is Logic Empirical?" In M. Dummett, Truth and Other Enigmas, pp. 269-289. London: Duckworth. (This chapter orig. publ. 1976.)

Dummett, Michael. 1991. The Logical Basis of Metaphysics. Cambridge, MA: Harvard University Press.

Dummett, Michael. 2000. Elements of Intuitionism, Second Edition. Oxford: Clarendon Press. (First edition 1977.)

Evans, Gareth. 1976. "Semantic Structure and Logical Form." In Truth and Meaning: Essays in Semantics, edited by G. Evans and J. McDowell, pp. 199-222. Oxford: Oxford University Press.

Evans, Gareth. 1978. 'Can There Be Vague Objects?' Analysis 38(4): 208. https://doi.org/10.1093/analys/38.4.208

Field, Hartry. 2008. Saving Truth from Paradox. Oxford: Oxford University Press.

Font, Josep M. 1999. "On Special Implicative Filters." Mathematical Logic Quarterly 45(1): 117-126. https://doi.org/10.1002/malq.19990450111

Font, Josep M. and Hájek, Petr. 2002. "On Łukasiewicz's Four-Valued Modal Logic." Studia Logica 70(2): 157-182. https://doi.org/10.1023/A:1015111314455

Font, Josep M., Jansana, Ramon and Pigozzi, Don. 2004. "A Survey of Abstract Algebraic Logic." Studia Logica 74 (2004), 13-97.

Gabbay, Dov M. 1993. "Classical vs Non-classical Logics: The Universality of Classical Logic." Technical Report MPI-I-93-230, Max-Planck-Institut Für Informatik, Saarbrücken.

Girard, Patrick and Weber, Zach. "Modal Logic Without Contraction in a Metatheory Without Contraction." Unpublished manuscript (2018).

Haack, Susan. 1976. "The Justification of Deduction." Mind 85(337) 112-119. https://doi.org/10.1093/mind/LXXXV.337.112

Halldén, Sören. "On the Semantic Non-completeness of Certain Lewis Calculi." Journal of Symbolic Logic 16(2): 127-129. https://doi.org/10.2307/2266686

Hawranek, Jacek. 1987. "On the Degree of Complexity of Sentential Logics, III. An Example of Johansson's Minimal Logic." Studia Logica 46(4): 283-289. https://doi .org/10.1007/BF00370640

Hawranek, Jacek and Zygmunt, Jan. 1981. "On the Degree of Complexity of Sentential Logics. A Couple of Examples." Studia Logica 40(2): 141-153. https://doi.org/10.1007/BF01874705

Hughes, George E. and Cresswell, Max J. An Introduction to Modal Logic. London: Methuen. 
Hughes, George E. and Cresswell, Max J. A New Introduction to Modal Logic. London: Routledge.

Humberstone, Lloyd. 1988. "Extensionality in Sentence Position." Journal of Philosophical Logic 15(1): 27-54; erratum ibid. 17(3): 221-223. "The Lattice of Extensional Connectives: A Correction." https://doi.org/10.1007/BF00250548

Humberstone, Lloyd. 1991. Review of Read 1988, Australasian Journal of Philosophy 69(2): 234-236. https://doi.org/10.1080/00048409112344681

Humberstone, Lloyd. 1996. "Homophony, Validity, Modality." In Logic and Reality: Essays on the Legacy of A. N. Prior, edited by B. J. Copeland, pp. 215-236. Oxford: Clarendon Press.

Humberstone, Lloyd. 2005. "Modality." In The Oxford Handbook of Contemporary Philosophy, edited by F. C. Jackson and M. Smith, pp. 534-614. Oxford: Oxford University Press.

Humberstone, Lloyd. 2006. "Extensions of Intuitionistic Logic Without the Deduction Theorem." Reports on Mathematical Logic 40: 45-82.

Humberstone, Lloyd. 2007a. "Logical Discrimination." In Logica Universalis: Towards a General Theory of Logic, edited by J.-Y. Béziau, pp. 225-46. Basel: Birkhäuser.

Humberstone, Lloyd. 2007b. "Modal Logic for Other-World Agnostics: Neutrality and Halldén Incompleteness." Journal of Philosophical Logic 36(1): 1-32. 10.1007/s10992-005-9020-9

Humberstone, Lloyd. 2008. "Replacing Modus Ponens With One-Premiss Rules." Logic Journal of the IGPL 16(5): 431-451. https://doi.org/10.1093/jigpal/jzn017

Humberstone, Lloyd. 2010. "Smiley's Distinction Between Rules of Inference and Rules of Proof." In The Force of Argument: Essays in Honor of Timothy Smiley, edited by J. Lear and A. Oliver, pp. 107-126. New York: Routledge.

Humberstone, Lloyd. 2011. The Connectives. Cambridge, MA: MIT Press. https://doi.org/10.7551/mitpress/9055.001.0001

Humberstone, Lloyd. 2016. Philosophical Applications of Modal Logic, College Publications, London.

Humberstone, Lloyd. Forthcoming. "Priest on Negation." In Graham Priest on Dialetheism and Paraconsistency, edited by C. Baskent and T. Ferguson.

Irvine, Andrew. 2013. "S7." Journal of Applied Logic 11(4): 523-529. https://doi.org/10.1016/j.jal.2013.07.001

Kostrzycka, Zofia. 2015. "On Halldén Completeness of Modal Logics Determined by Homogeneous Kripke Frames." Bulletin of the Section of Logic 44(3-4): 111-130. http://dx.doi.org/10.18778/0138-0680.44.3.4.02

Kracht, Marcus. 1999. Tools and Techniques in Modal Logic. Amsterdam: North-Holland (Elsevier).

Kreisel, Georg. 1958. "A Remark on Free Choice Sequences and the Topological Completeness Proofs." Journal of Symbolic Logic 23(4): 369-388. https://doi.org/10.2307/2964012

Organon F 26 (3) 2019: 340-404 
Kreisel, Georg. 1962. "On Weak Completeness of Intuitionistic Logic." Journal of Symbolic Logic 27(2): 139-158. https://doi.org/10.2307/2964110

Kremer, Philip. 1999. "Relevant Identity." Journal of Philosophical Logic 28(2): 199-222. https://doi.org/10.1023/A:1004323917968

Kripke, Saul A. 1965. "Semantical Analysis of Modal Logic II. Non-Normal Modal Propositional Calculi." In The Theory of Models, edited by J. W. Addison, L. Henkin, and A. Tarski, pp. 206-220. Amsterdam: North Holland.

Lemmon, Edward J. 1966. "A Note on Halldén-Incompleteness." Notre Dame Journal of Formal Logic 7(4): 296-300. doi:10.1305/ndjfl/1093958745

Lewis, Clarence Irving and Langford, Cooper Harold. 1959. Symbolic Logic. New York: Dover. (Originally publ. 1932, without Appendix III.)

Łoś, Jerzy. 1955. "The Algebraic Treatment of the Methodology of Elementary Deductive Systems." Studia Logica 2: 151-211. https://doi.org/10.1007/BF02124771

Łoś, Jerzy and Suszko, Roman. 1955. "On the Extending of Models, II." Fundamenta Mathematicae 42: 343-347. https://doi.org/10.4064/fm-42-2-343-347

Łoś, Jerzy and Suszko, Roman. 1958. "Remarks on Sentential Logics." Indagationes Mathematicae 20: 177-183.

Makinson, David. 1971. "Some Embedding Theorems for Modal Logics." Notre Dame Journal of Formal Logic 12(2): 252-254. https://doi.org/10.1305/ndjfl/1093894226

Makinson, David. 1973. "A Warning About the Choice of Primitive Operators in Modal Logic." Journal of Philosophical Logic 2(2): 193-196.

Mares, Edwin D. 1992. 'Semantics for Relevance Logic with Identity', Studia Logica 51(1): 1-20. https://doi.org/10.1007/BF00370329

Mares, Edwin D. 2003. 'Halldén-completeness and Modal Relevant Logic', Logique et Analyse 46: 59-76.

Mason, Ian. 1985. 'The Metatheory of the Classical Propositional Calculus is Not Axiomatizable', Journal of Symbolic Logic 50(2): 451-457.

https://doi.org/10.2307/2274233

McCarty, Charles. 2008. "Completeness and Incompleteness for Intuitionistic Logic." Journal of Symbolic Logic 73(4): 1315-1327. https://doi.org/10.2178/jsl/1230396921

McKinsey, J. C. C. 1953. 'Systems of Modal Logic Which are Not Unreasonable in the Sense of Halldén', Journal of Symbolic Logic 18(2): 109-113. https://doi .org/10.2307/2268941

Meyer, Robert K. 1985. Proving Semantic Completeness "Relevantly" for R, Logic Research paper No. 32, Logic Group, Department of Philosophy, RSSS, Australian National University.

Meyer, Robert K. and Martin, Errol P. 1986. "Logic on the Australian Plan." Journal of Philosophical Logic 15(3): 305-332. https://doi.org/10.1007/BF00248574

Meyer, Robert K. and Routley, Richard. 1977. "Extensional Reduction I." The Monist 60(3): 355-369. https://doi.org/10.5840/monist197760316 
Miura, Satoshi. 1966. "A Remark on the Intersection of Two Logics.', Nagoya Mathematical Journal 26: 167-171.

Pledger, K. E. 1972. "Modalities of Systems Containing S3." Zeitschr. für math. Logik und Grundlagen der Math. 18: 287-283.

Porte, Jean. 1979. "The $\Omega$-System and the E-System of Modal Logic." Notre Dame Journal of Formal Logic 20(4) 915-920.

Priest, Graham. 1984. "Logic of Paradox Revisited." Journal of Philosophical Logic 13(2): 153-179. https://doi.org/10.1007/BF00453020

Priest, Graham. 2006. Doubt Truth to be a Liar. Oxford: Oxford University Press.

Prior, Arthur N. 1956. "Logicians at Play; Or Syll, Simp, and Hilbert." Australasian Journal of Philosophy 34(3): 182-192. https://doi.org/10.1080/00048405685200181

Prior, Arthur N. 1957. Time and Modality. Oxford: Oxford University Press.

Putnam, Hilary. "Vagueness and Alternative Logic." Erkenntnis 19(1-3): 297-314. https://doi.org/10.1007/BF00174788

Rasiowa, Helena. 1974. An Algebraic Approach to Non-Classical Logics. Amsterdam: North-Holland.

Read, Stephen. 1988. Relevant Logic: A Philosophical Examination of Inference. Oxford: Blackwell.

Routley, Richard and Meyer, Robert K. 1972. "The Semantics of Entailment - II." Journal of Philosophical Logic 1(1): 53-73. https://doi.org/10.1007/BF00649991

Routley, Richard, Plumwood, Val, Meyer, Robert K. and Brady, Ross T. 1982. Relevant Logics and their Rivals. Atascadero: Ridgeview.

Schumm, George F. 1969. "On Some Open Questions of B. Sobociński." Notre Dame Journal of Formal Logic 10(3): 2610-261. https://doi.org/10.1305/ndjfl/1093893711

Schumm, George F. 1975. "Disjunctive Extensions of S4 and a Conjecture of Goldblatt's." Zeitschr. für math. Logik und Grundlagen der Math. 21: 81-86.

Schumm, George F. 1993a. "Halldén Complete Modal Logics that are not Strongly Incomplete." Bulletin of the Section of Logic 22: 158-160.

Schumm, George F. 1993b. "Why Does Halldén-Completeness Matter?" Theoria 59(1): 192-206. https://doi.org/10.1111/j.1755-2567.1993.tb00870.x

Segerberg, Krister. 1968. "Propositional Logics Related to Heyting's and Johansson's." Theoria 34(1): 26-61. 10.1111/j.1755-2567.1968.tb00337.x

Segerberg, Krister. 1975. "That Every Extension of S4.3 is Normal." In Procs. Third Scandinavian Logic Symposium, edited by S. Kanger, pp. 194-196.

Shapiro, Stewart. 2014. Varieties of Logic. Oxford: Oxford University Press.

Shoesmith, D. J. and Smiley, Timothy J. 1971. "Deducibility and Many-Valuedness." Journal of Symbolic Logic 36(3): 610-622. https://doi.org/10.2307/2272897

Shoesmith, D. J. and Smiley, Timothy J. 1978. Multiple-Conclusion Logic. Cambridge: Cambridge University Press.

Organon F 26 (3) 2019: 340-404 
Shukla, Anjan. 1972. "The Existence Postulate and Non-Regular Systems of Modal Logic." Notre Dame Journal of Formal Logic 13(3): 369-378.

https://doi .org/10.1305/ndjfl/1093890624

Sobociński, Bołeslaw. 1964. "A Note on Prior's Systems in 'The Theory Of Deduction'." Notre Dame Journal of Formal Logic 5(2): 139-140.

https://doi.org/10.1305/ndjfl/1093957805

Sotirov, Vladimir. 2008 "Non-Classical Operations Hidden in Classical Logic." Journal of Applied Non-Classical Logics 18(2-3): 309-324. https://doi.org/10.3166/jancl.18.309-324

Stairs, Allen. 2016. "Could Logic be Empirical? The Putnam-Kripke Debate." In Logic and Algebraic Structures in Quantum Computing, edited by J. Chubb, A. Eskandarian and V. S. Harizanov, pp. 23-4. Cambridge: Cambridge University Press.

Suszko, Roman. 1971. "Quasi-completeness in Non-Fregean Logic." Studia Logica 29(1): 7-14. https://doi.org/10.1007/BF02121851

Suszko, Roman. 1975. "Abolition of the Fregean Axiom." In Logic Colloquium, edited by R. Parikh, pp. 169-239. Berllin: Springer.

Tennant, Neil. 2005. "Relevance in Reasoning." In The Oxford Handbook of Philosophy of Mathematics and Logic, edited by S. Shapiro, pp. 696-726. Oxford: Oxford University Press.

Weber, Zach, Badia, Guillermo and Girard, Patrick. 2016. "What is an Inconsistent Truth Table?" Australasian Journal of Philosophy 94(3): 533-548. https: //doi .org/10.1080/00048402.2015.1093010

Williamson, Timothy. 2011. "Logics and Metalogics." In Logic and Knowledge, edited by C. Cellucci, E. Grosholz and E. Ippoliti, pp. 81-107. Newcastle: Cambridge Scholarly Publishing.

Williamson, Timothy. 2013. Modal Logic as Metaphysics. Oxford: Oxford University Press.

Williamson, Timothy. 2014. "Logic, Metalogic and Neutrality." Erkenntnis 79(2): 211-231. https://doi .org/10.1007/s10670-013-9474-z

Williamson, Timothy. 2017. "Dummett on the Relation between Logics and Metalogics." In Truth, Meaning, Justification, and Reality: Themes from Dummett, edited by M. Frauchiger, pp. 153-175. Berlin: Walter de Gruyter.

Wójcicki, Ryszard. 1974. "Note on Deducibility and Many-Valuedness." Journal of Symbolic Logic 39(3): 563-566. https://doi.org/10.2307/2272897 


\section{Appendix: longer notes for sections 1-3}

\section{For section 1}

On Dummett and others. Although here we are mainly concerned with the homophonic case, it is worth recording some contrasting reactions to nonhomophonic (though still isological) model theory alluded to in Section 1. At p. 331 of (Brady 2003), we read:

In more recent times, even the study of formal logics, not based on classical logic and seen as alternatives to classical meta-logic has been pursued using a classical logic, with little if any explanation of this, thus creating a conflict between the object- and metalogics. This has certainly been the case with relevant logics and intuitionistic logic, with very little written on the use of these non-classical logics in setting up their own meta-theory. (But, see Meyer 1985.)

The publication date for this source is 2003 though of course there was a considerable gestation period for some of the material in (Brady 2003), so it is interesting to see a rather different view of how "very little" work has been done in the direction in question coming from (Dummett 1991, 54f.), a passage quoted in (Williamson 2011) and (Williamson 2017):

A thoroughly pernicious principle has gained considerable popularity in recent years. It is that in formulating a semantic theory the metalanguage must have the same underlying logic as the objectlanguage. When this principle is followed, the proponent of a nonclassical logic has a perfect counter to an argument in favour of a classical law that he rejects, namely, that the argument assumes the validity of the law in the metalanguage.

Williamson is not concerned with the issue of how prevalent such work has been, but with the merits of Dummett's proposed alternative methodology: that in discussing their respective logics with each other, the parties to the discussion (the classical and intuitionistic logician, for instance) should not exploit features on which they disagree, but set up any semantic apparatus in such a way as to be insensitive to that disagreement, allowing the discussion to 
proceed on neutral terms. ${ }^{42}$ This desire of Dummett's to couch the discussion in terms not contested by either party is presumably what leads him to include a condition on the treatment of sentence letters $p$ at a node $a$ in a Beth model for intuitionistic logic - condition $\left(\mathrm{iii}_{b}\right)$ on p. 139 of (Dummett 1977) (or p. 191 of the first edition) - to the effect that " $p$ is verified at $a$ or $p$ is not verified at $a$ ", whose inclusion will strike classically backgrounded readers as problematic not because it introduces something potentially controversial but because it suddenly becomes evident that Dummett is not conducting the discussion in the usual 'classical meta-logic' style and explicitly imposing a condition which from that point of view would be vacuous, so that the reasoning will be acceptable to intuitionist. (This condition does not have the same unfortunate effects as Brady and Rush's condition $\left(^{*}\right)$ is seen to have in the present section, because Dummett is presenting Beth's far-from-homophonic model theory, but doing so while conducting the discussion using no intuitionistically contested metalogical principles. Thus Dummett often includes language such as the following - from p. 163 of Dummett 1977 - "if we wish our argument itself to be intuitionistically valid". )

Dummett's 'neutral ground' idea bears on the remarks quoted from (Priest 2006) in the main body of Section 2: "Is it to be supposed that their account of this behaviour is to be given in a way that they take to be incorrect? Clearly not. The same logic must be used in both 'object theory' and 'metatheory'." Taking "incorrect" to mean "not correct", there are two senses to bear in mind for such phrases as correct logic. One may have in mind a favoured account of validity w.r.t. which the logic in question is sound and complete, and by correct mean precisely that: both sound and complete. Alternatively, with such a notion of validity in mind one may simply mean that the logic is sound w.r.t. that notion of validity. When invited by Priest's rhetorical question as to whether someone should be expected to reason in accordance with a logic which is not correct, one is inclined to agree because one should not be

\footnotetext{
${ }^{42}$ (Aberdein and Read 2009, 649), touch on the passage quoted from Dummett with apparent approval, and also provide some pertinent further references. (Field 2008, 111-114), also stresses Dummett's desire not to beg contested questions, though he seems more confident than Dummett that a classical metatheory achieves the required neutrality. Here is Field on p. 111: "Suppose (...) that an advocate of a non-classical logic for vagueness were to propose a definition of validity for vague languages in vague terms. In that case, one would presumably have to use that non-classical logic to apply the definition. Most people who advocated other logics, e.g. classical logic, would protest that they didn't know what to make of the proposed logic: that when they employed the definition, using their own classical reasoning, they got different results from the non-classical logician who advocated the definition. The point of a definition of validity in an effectively classical set theory that virtually all theorists can agree on is precisely to avoid such an unpleasant situation."
} 
expected to reason unsoundly by one's own lights. But this requires taking "correct" in the second of the two senses just distinguished. There is no similar pressure to answer, as Priest does "Clearly not", if correctness is taken the first way: there is nothing wrong with being asked (for dialectical purposes) to reason according to a logic which is weaker than one that is complete according to one's favoured notion of validity, and so we cannot conclude without further ado, as Priest does here, that the same logic must be used at the two levels. (However, the 'further ado' is readily forthcoming in Priest's case, since the logic - classical logic - he is being expected to reason in accordance with does endorse principles, such as disjunctive syllogism, which he rejects, rather than merely being expected to refrain from availing himself of logical principles he accepts.)

Williamson's discussion of Dummett, to resume that thread, follows Dummett's own example of proceeding, next, to consider the case of quantum logic in which a homophonic treatment of conjunction and disjunction - exactly the connectives for which such a treatment was unproblematic intuitionistically and relevantly - would be an evident violation of Dummett's injunction to try to couch the semantical discussion in terms which do not immediately beg the question as to whether the contested distribution laws are correct. Dummett has long stressed (e.g., in Dummett 1976, 274f.), approvingly reporting some thoughts of Putnam's, that the use of truth-tables to settle difference between this pair of parties violates the insensitivity demand in its presumption that the $2^{k}$ rows (where $k$ sentence letters are involved) exhaust the cases to be considered. (The much less enthusiastic reception given to Putnam's ideas by Kripke here is reported in pp. 192-199 of Berger 2011; see also Stairs 2016.) Isn't it odd, though, that the parties to this debate entitle their papers "Is logic empirical?" on the grounds that the facts of quantum mechanics are taken, by those returning an affirmative answer to this question, as evidence against the correctness of the $\Lambda / \vee$ distribution laws? After all, even if the quantum mechanical facts had been otherwise, defenders of this answer would presumably believe they could instead have been as currently envisaged, so the distribution laws would not be necessarily or a priori correct. And this mere possibility is not something that needs to be established empirically, even if it is the empirical facts that (according to Putnam and others) bring it to our attention as an actualized possibility. 
Soundness without reference to a particular axiomatization. To conclude the initial puzzles raised in Section 1 by the Brady-Rush passage, one further issue calls for comment. The question of soundness and completeness usually arises when, with a notion of validity to hand, provided by whatever semantic account is in play, one wants to compare it with provability in this or that proof-system, such as an axiomatic system, a natural deduction system, or a sequent calculus. But there is no mention, even in general terms, in the Brady-Rush discussion, of any axioms or rules, to take the axiomatic route (suggested by their talking of completeness as requiring us to show that an arbitrary nontheorem is not valid, rather than, more generally, that an unprovable sequent is not valid). Though we will have a look at how some of this would go for a concrete axiomatization of a propositional logic in Section 2 , it should be acknowledged that the Brady-Rush discussion can be made sense of without the consideration of proof system: we simply take the set of (in the axiomatic case) theorems as given: this is the logic we are to deal with and to show its soundness is to show that all of them are valid, and for its completeness that no other formulas of the language in question are valid. This is the course explicitly adopted in (Bacon 2013), for example, whose treatment (p. 347) helps itself "by brute force" to a predicate Prov of formulas and axioms in the (non-classical) formal metatheory $\operatorname{Prov}(A)$ for each theorem $A$ of the object logic and (what we may think of as) the negation of $\operatorname{Prov}(A)$ for non-theorem, as well as inductive axioms like those concerning the connectives in (Brady and Rush 2009). ${ }^{43}$ Further, while Brady and Rush discuss arbitrary logics (concentrating on arbitrary non-classical logics but presumably not intending to exempt classical logic from the claimed triviality of the procedure they describe - see the last paragraph of Section 3), Bacon delimits algebraically a range of logics, essentially Rasiowa's 'standard systems of implicative extensional propositional calculi' (to quote the title of Rasiowa $1974, \S 8.5)$, and - a definite bonus - stresses logics as consequence relations (or suitable sets of sequents, we may say) rather than sets of formulas, for consideration in (Bacon 2013): ${ }^{44}$ each such logic is the consequence relation

\footnotetext{
${ }^{43}$ Bacon writes "Prov $(\ulcorner\varphi\urcorner)$ " rather than " $\operatorname{Prov}(A)$ " and has a slightly different way of dealing with the sentence letters than Brady and Rush. The full sentence in which the phrase "brute force" appears is as follows: "Here this done by brute force - a thorough metatheorist might wish to set up a classical theory of syntax and provide an axiomatic account of provability." An example of such thoroughness, devoted to the case of classical propositional logic, is perhaps provided by (Mason 1985).

${ }^{44}$ Throughout the discussion consequence relations are to be understood in accordance with the standard definition, as given for example in (Kracht 1999), §1.4; when considered as logics, we take them to be specif-
} 
determined by which is given a matrix based a complete lattice (top element designated) equipped with a binary implication-interpreting operation connective satisfying the conditions of (Rasiowa 1974) defining what are there (Chapter 2) called implicative algebras. ${ }^{45}$

\section{For section 2}

Brady on the interpretational meta-logic. On turning, as instructed in the quotation in Section 2 from (Brady and Rush 2009), to Chapter 13 of (Brady 2003), one finds a section entitled 'Interpretational Meta-Logic and the Semantics of Quantified Relevance', with the following words by way of explanation of this distinction (p.332); the first ellipsis in this quotation recalls such conditions - numbered (i), (ii), (iii) in (Brady 2003) - as those for $\rightarrow$, \&, etc., under (2) of the quoted passage in Section 1 :

When one thinks about it, there is a part of the meta-theory that corresponds most closely to the object-logic, that is, the logic of the meta-logical connectives used in expressing the interpretation statements (...) A classical meta-logic could be built up from just these meta-logical connectives, as it is part of the Hilbertian usage of meta-logic. This meta-logic could reasonably be seen as the interpretation of the corresponding object-logic and thus we will call it the interpretational meta-logic. This is just the logic of the interpretation statements, without reference to the underlying meta-logic, which we take to be the logic used to set up the rest

ically substitution-invariant consequence relations - satisfying the condition (sub.) in Kracht's discussion, that is - and all examples in the present paper are also finitary (satisfy Kracht's condition (cmp.), that is).

${ }^{45}$ The logical principles concerning the implicational connective, for which we write $\rightarrow$, thereby secured are: $A, A \rightarrow B \vdash B ; \vdash A \rightarrow A ; A \vdash B \rightarrow A$ (evidently a principle which would not appeal to Brady and Rush, given their relevant-logical sympathies); $A \rightarrow B, B \rightarrow C \vdash A \rightarrow C$; and $A \rightarrow B, B \rightarrow A \vdash C(A) \rightarrow C(B)$ where $C(A), C(B)$ are formulas resulting from uniformly substituting resp. $A, B$ for the same sentence letter occurring in some formula $C$. If that sentence letter is, say $p$, one would often more explicitly write $C$ as $C(p)$, but since its identity is immaterial, the neutral notation $C(\cdot)$ is more suggestive and is used in the main body of Section 2. (See Chapter 8 of Rasiowa 1974 for details, and Font 1999 for corrections of some aspects of Chapters 1 and 8 of Rasiowa 1974.) Returning to Bacon, the binary meets and joins in the lattice interpret conjunction and disjunction connectives, with their potentially infinitary cousins interpreting quantifiers. The latter also provide top and bottom elements, the latter being used as the consequent of an implication to interpret negation (slightly oversimplifying the actual details of Bacon's presentation here, which in fact includes not only first-order quantifiers but monadic second-order quantifiers, the latter used for quantifying over models in the setting in which all we need to know about a model is which atomic formulas it verifies, and so models can be identified with sets or properties of such formulas).

Organon F 26 (3) 2019: 340-404 
of the semantics. Moreover, for some suitable purpose, we could separate off this interpretational meta-logic from the underlying meta-logic, even though both logics are classical. Since the interpretational meta-logic satisfies (i), (ii) and (iii), it is indeed an applied classical logic, applied to interpretation statements of the form $I(A)=T$ (or $F$ ), with (i)-(iii) acting as meaning postulates which determine the interaction of the meta-logical connectives with these statements. (Note that the 'iff's in (i)-(iii) are of the underlying meta-logic.)

Although Brady supplies several further paragraphs in elaboration of this distinction, which broaches the subject of the quasi-homophonic version of the connectives in question, the idle parameters being for points in a RoutleyMeyer model, the present author was able to make little better sense of them than of the above passage and the interested reader is directed to that discussion (Brady 2003, 332-334). The reason for including the above passage is the fact that Brady and Rush refer to it in (Brady and Rush 2009) as background - despite the difference in orientation ("both logics are classical"46) and because of the last parenthetical sentence: the occurrences of 'iff' are part of the (language of) the underlying meta-logic.

Even if it is not be entirely clear what Brady has in mind, this passage does at least allow us to conclude that the occurrences of "iff" in the conditions the Section 1 passage need not correspond to a (primitive or defined) connective of the object language. Thus in what may seem to be cavalier disregard for the contrast for the problematic distinction between interpretational and underlying meta-logics here - if this is anything other than the contrast between homophonic and non-homophonic semantic theories in the single meta-logic in play - we take it that the metalanguage will typically have additional logical vocabulary governed by the (one and only) meta-logic in accord with which the semantics is being discussed, over and above the logical vocabulary of the object language, alongside the quantifiers already granted that status in Section 1 (for the semantics of a propositional logic).

Bacon and others. As was mentioned in the second of the longer notes on Section 1, (Bacon 2013) is not concerned to show the soundness of a

\footnotetext{
${ }^{46}$ In which case, one might ask, why are we talking about two logics? And the logics differ how do they get to rub shoulders together in reasoning to a verdict of validity or invalidity of some candidate formula or inference in the object language, for instance?
} 
particular axiomatization of a logic within the (algebraically delimited) range with which but just with showing that all the provable formulas - however they get to be provable - are valid, where this last is a matter of truth on every interpretation much as in Brady and Rush's discussion. The validity and provability of a formula $A$ are expressed in the metalanguage by $\operatorname{Prov}(A)$ and $\operatorname{Valid}(A) .{ }^{47}$ He calls the semantic theory in this style for a logic $L, T_{L}-$ so Prov and Valid mean, respectively, provability in $L$ and validity according to $T_{L}$ - and describes $L$ as weakly sound w.r.t. $T_{L}$ if (quoting from the formulation in Coro. 2.3 of Bacon 2013, ${ }^{48}$ changing " $\varphi$ " to " $A$ ") "Valid $(A)$ follows in $L$ from $T_{L} \cup\{\operatorname{Prov}(A)\}$ ", which in an adaptation of the notation used in the body of Section 2 would be written as

$$
T_{L}, \operatorname{Prov}(A) 上_{L} \operatorname{Valid}(A),
$$

with $T_{L}$ playing the same kind of ancillary role as " $(\rightarrow / \rightrightarrows)$ " in $(\mathrm{mp})$ in Section 2, and which Bacon distinguishes from strong soundness:

$$
T_{L} 上_{L} \operatorname{Prov}(A) \rightrightarrows \operatorname{Valid}(A) .
$$

Note that this strengthening of soundness has nothing to do with the strengthening alluded to in the discussion of $\vdash_{B C K}$ in Section 2 which involves allowing $n>0$ in the claim that $A_{1}, \ldots, A_{n} \vdash_{B C K} B$ implied the truth of $B$ on every interpretation verifying each of the $A_{i}$. Bacon argues strong soundness for all $L$ in his range, according to the corresponding $T_{L}$, on the basis of weak soundness of aspects of his $T_{L}$ not gone into in this summary, rather than on the basis of the deduction theorem for $L$, which he notes not all logics in the range satisfy - the case of $\vdash_{B C K}$ (and thus $上_{B C K}$ ) considered here being one such case. $^{49}$ ( (Girard and Weber 2018) also take as their basic logic $B C K$, with quantifiers added as well as multiplicative or intensional conjunction - see note 7 - but additive or extensional disjunction, on the other hand, and add modal operators with a view to treating them semantically using the

\footnotetext{
${ }^{47}$ Bacon actually uses letters from the range $\varphi, \psi$, puts corner-quotes around them for talking about them in the metalanguage.

${ }^{48}$ I quote the Corollary here rather than the actual definition(s) at the start of Section 2.3 of (Bacon 2013), since weak soundness and completeness are given each other's definitions by mistake (as I am grateful to Bacon for confirming for me).

${ }^{49} \mathrm{~A}$ famous counterexample here: $p \rightarrow(p \rightarrow q), p \vdash_{B C K} q$ while $p \rightarrow(p \rightarrow q) \nvdash_{B C K} p \rightarrow q$; there are of course more general 'parametrized local' versions of the deduction theorem which are satisfies in such cases, that of $\vdash_{B C K}$ being reviewed in Example 2.1.2 of (Czelakowski 2001) - or Corollary 1.29.10 in (Humberstone 2011) for a range of cases.
}

Organon F 26 (3) 2019: 340-404 
Kripke model-theoretic truth-definition in the modal-free part of an analogous metalogic, paying special attention to the complications caused by the lack of contraction for this enterprise.) While on the subject of soundness, mention should be made of (Weber, Badia and Girard 2016), in which a paraconsistent metalogic is used in the semantic metatheory, the latter theory itself being inconsistent - and proudly so - with the authors establishing to their own satisfaction at least (p. 542) that the object logic is sound and not sound (as well as that the law of excluded middle is both valid and invalid). Hmm...

\section{For section 3}

Bell and others. What follows is a passage from p.6f. of (Bell 1988), discussing in a free and easy way, which is nevertheless insistently intuitionistic in its logic, an aspect of the models ('smooth worlds') of the (smooth) infinitesimal analysis that the book - to which the attention of the philosophyof-logic community has recently been drawn by (Shapiro 2014) - is all about; in the passage quoted $\mathbb{S}$ stands for such a 'smooth world', and the use of $I$ (for Infinitesimals) has nothing to do with its use elsewhere in our discussion for interpretations:

If we now call two points $a, b$ on the real line distinguishable or distinct when they are not identical, i.e., not $a=b-$ which as usual we write as $a \neq b$ - and indistinguishable in the contrary case, i.e., if not $a \neq b$, then in $\mathbb{S}$, indistinguishability of points will not imply their identity. As a result, the 'infinitesimal neighbourhood of 0' comprising all points indistinguishable from 0 - which we will denote by $I$ - will, in $\mathbb{S}$, be nonpunctiform in the sense that it does not reduce to $\{0\}$, that is,

it is not the case that 0 is the sole member of $I$.

If we call the members of I infinitesimals, then this statement may be rephrased:

it is not the case that all infinitesimals coincide with 0 .

Observe, however, that we cannot go on from this to infer that there exists an infinitesimal which is $\neq 0$. 
For such an entity would possess the property of being both distinguishable and indistinguishable from 0 , which is clearly impossible.

If being the sole member of $I$, as in the first line inset here, is being the only thing that is an element of $I$ then the three representations canvassed in the main body of Section 3 for "Only $F$ s are $G$ " would be, in order: (i) $\forall x(x \in$ $I \rightarrow x=0$ ), (ii) $\forall x(\neg x=0 \rightarrow \neg x \in I)$, and (iii) $\neg \exists x(x \in I \wedge \neg x=0$ ). It seems clear from the gloss Bell gives in the following inset as a rephrasing follows the structure of the negation of (i), and presumably more generally takes " $a \in\left\{b_{1}, \ldots, b_{n}\right\}$ " to amount to " $a=b_{1} \vee \ldots \vee a=b_{n}$ ". Compare now, (Bacon 2013), discussing a logic for vagueness which is like intuitionistic logic in at least this respect: it does not endorse the law of excluded middle. ${ }^{50}$ At p. 342 there Bacon has us consider a function $v$, defined in deliberately vague by saying that $v\left(P_{i}\right)=1$ if $i$ is small and $v\left(P_{i}\right)=0$ otherwise, adding that " $v$ here is 'bivalent' in the sense that the codomain of $v$ consists of two truth values: $\{0,1\}$." To this is appended a footnote (note 7 ) which reads

To say that $D$ is the codomain of a function $f$ is to say that $\forall x(\exists y(f(y)=x \rightarrow x \in D))$. In the logics considered the codomain of $v$ being $\{0,1\}$ does not entail that $v(x)=1 \vee v(x)=0$ for any $x$.

Presumably this last means (eliminating an ambiguity in "any" here): "does not entail that for all $x v(x)=1 \vee v(x)=0$ ", though the significance of writing " $\forall x(\exists y(f(y)=x \rightarrow x \in D))$ " rather than simply " $\forall y(f(y) \in D)$ " is not immediately clear, ${ }^{51}$ but taking this simpler formulation as adequate the attention may be to say that, recalling that $D$ is, in the case under consideration, $\{0,1\}, y \in\{0,1\}$ does not mean, as suggested above for Bell, $y=0 \vee y=1$ but rather $\neg(y \neq a \wedge \neg y \neq b \wedge \neg y \neq c)$. Bacon himself may not quite have intuitionistic logic in mind for vagueness, though the suggestion is occasionally made (e.g., Putnam 1983) and this gives a weaker notion of set membership than that we presumed was operative in Bell's discussion, serving again to

\footnotetext{
${ }^{50}$ On p. 104 in a chapter of (Bell 1988) making the logic explicit, the status of the law of excluded middle in the theory of smooth infinitesimal analysis is explained in more detail by Bell: (i) the theory proves the negations of some universally quantified 'excluded middles', rather than simply failing to prove the quantified forms, and (ii) in "most models" of the theory all disjunctions $A \vee \sim A$ are true when $A$ is a closed formula.

${ }^{51}$ Bacon has offered (p.c.) some clarification: function symbols are not officially part of the language he is using here and " $f(x)=y$ " is shorthand for " $\langle x, y\rangle \in f$ ", with " $f(x) \in\{0,1\}$ " " short for something along the lines of like " $\exists y((x, y) \in f \wedge y \in\{0,1\})$ " or " $\forall y((x, y) \in f \rightarrow y \in\{0,1\})$ ".
}

Organon F 26 (3) 2019: 340-404 
illustrate the challenging multiplicity of interpretations weakening one's logic can induce, to say nothing of the awkwardness of having to figure out exactly when a transition from $\neg A \rightarrow B$ to $A \vee B$ and thence to some further conclusion can be reformulated (still terminating at that further conclusion) so as to keep one's reasoning acceptable by the lights of intuitionistic logic, and similarly with the case of the converse transition in the case of relevant logic. ${ }^{52}$

\footnotetext{
${ }^{52}$ This leads us in Section 4 to explore options - such as classically based modal logics - that retain classical (non-modal) logic, in the interests of making things easier to think through, rather than out of some conviction of the all-purpose superiority (cf. Gabbay 1993). And although it is sometimes hard to say when this or that classical principle is essential to a piece of reasoning, it is not always hard: see the index entries under "choice of meta-logic" in (Humberstone 2011), for instance, as well as such discussions as those of (Bell 1988).
}

Organon F 26 (3) 2019: 340-404 